\title{
Dynamically tunable multiband plasmon-induced transparency effect based on graphene nanoribbon waveguide coupled with rectangle cavities system
}

Zihao Zhu

Hubei Engineering University

Boyun Wang ( $\nabla$ wangboyun@alumni.hust.edu.cn )

Hubei Engineering University https://orcid.org/0000-0001-5293-0734

Xiang Yan

Hubei Engineering University

Yang Liu

Hubei Engineering University

Qingdong Zeng

Hubei Engineering University

\section{Tao Wang}

Hubei Engineering University

Huaqing Yu

Hubei Engineering University

\section{Research Article}

Keywords: Plasmon-induced transparency, Plasmonic waveguide, Graphene, Finite difference time domain, Group index, Rectangle cavities

Posted Date: February 3rd, 2022

DOI: https://doi.org/10.21203/rs.3.rs-1282709/v1

License: (c) (1) This work is licensed under a Creative Commons Attribution 4.0 International License.

Read Full License 


\title{
Dynamically tunable multiband plasmon-induced transparency effect based on graphene nanoribbon waveguide coupled with rectangle cavities system
}

\author{
Zihao Zhu ${ }^{1} \cdot$ Boyun Wang ${ }^{1,2 *} \cdot$ Xiang Yan ${ }^{1} \cdot$ Yang Liu $^{1} \cdot$ Qingdong Zeng $^{1} \cdot$ Tao Wang $^{2} \cdot$ Huaqing Yu \\ ${ }^{1}$ School of Physics and Electronic-information Engineering, Hubei Engineering University, Xiaogan 432000, People's \\ Republic of China \\ ${ }^{2}$ Wuhan National Laboratory for Optoelectronics, Huazhong University of Science and Technology, Wuhan 430074, \\ People's Republic of China \\ *E-mail: wangboyun@alumni.hust.edu.cn \\ Zihao Zhu and Boyun Wang are co-first authors of the article.
}

\begin{abstract}
A dynamically tunable multiband plasmon-induced transparency (PIT) effect in a series of rectangle cavities coupled with a graphene nanoribbon waveguide system is investigated theoretically and numerically by tuning the chemical potential of the graphene rectangle cavity. A single-PIT effect is realized using two different methods: one is the direct destructive interference between bright and dark modes, and the other is the indirect coupling through a graphene nanoribbon waveguide. Moreover, dual-PIT effect is obtained by three rectangle cavities side-coupled with a graphene nanoribbon waveguide. Results show that the magnitude of the dual-PIT window can be controlled between 0.21 and 0.74 , and the corresponding group index is controlled between 143.2 and 108.6. Furthermore, the triple-PIT effect is achieved by the combination of bright-dark mode coupling and the cavities side-coupled with waveguide mechanism. Thus, sharp PIT windows can be formed, a high transmission is maintained between 0.51 and 0.74 , and the corresponding group index is controlled between 161.4 and 115.8. Compared with previously proposed graphene-based PIT effects, the size of the introduced structure is less than $0.5 \mu \mathrm{m}^{2}$. Particularly, the slow light effect is crucial in the current research. Therefore, a novel approach is introduced toward the realization of optical sensors, optical filters, and slow light and light storage devices with ultra-compact, multiband, and dynamic tunable.
\end{abstract}

Keywords Plasmon-induced transparency $\cdot$ Plasmonic waveguide $\cdot$ Graphene $\cdot$ Finite difference time domain $\cdot$ Group index $\cdot$ Rectangle cavities

\section{Introduction}

Electromagnetically induced transparency (EIT) effect [1, 2], a quantum effect, which is caused by the destructive interference between the two different pathways excited in the so-called three-level atomic systems [2], has shown various potential applications in fields, such as slowing light [3, 4], light storage [5], and optical filtering [6]. However, effective nonlinear materials and rigorous conditions are difficult to find, thereby significantly limiting the integrated application in practical optical devices. However, the plasmon-induced transparency (PIT) effect obtained by the cavity coupled with a plasmonic waveguide systems has caused extensive attention on account of simple realization. The devices realizing the PIT effect can be fabricated with a small footprint because surface plasmon 
polaritons (SPPs) can support deep subwavelength scale modes and overcome classical diffraction limit [7-9]. At present, diversified structures of PIT effect realization have been proposed. Moreover, the cavities side-coupled with a waveguide and the bright-dark mode coupling methods are of special guidance for generating a PIT window in various structures [10-13]; these methods are beneficial to onchip integration plasmonic devices, thereby facilitating easy fabrication of an ultra-compact structure.

Thus far, many plasmonic devices for realizing PIT effect have been investigated, and the cavities coupled with a metal-dielectric-metal (MDM) plasmonic waveguide is particularly prominent in highly integrated devices. Moreover, the coupling of MDM waveguides with double U-cavity [14], double-stub cavities [15], and double-disk cavities [16] have a great deal of research in the area of integrated slow light devices. However, only structural parameters can be adjusted once the devices are designed. Thus, obtaining dynamically tunable PIT effects at a specific wavelength is difficult. Graphene, a novel 2D material for tunable optical devices, comprises a single layer of carbon atoms; it has been recently widely considered by researchers due to its advantages of dynamic tunability, strong locality, and low loss [17]. The chemical potential of graphene can be flexibly controlled by applying varying applied voltage. Therefore, this feature provides graphene with an advantage for new tunable photonic device materials. Thus, many PIT devices made of graphene with excellent slow light properties and dynamic tunability, such as plasmonic waveguide coupled with nanocavities [18, 19], metamaterials [20-23], and metallic grating coupled with a dielectric waveguide layer (GCDWL) structures, have been proposed due to these unique performance advantages. Among of the various graphene structures, fabricating metamaterials and GCDWL structures through the current fabrication process is relatively complicated on account of the limitation of the micro machining etching accuracy [24]. Furthermore, integrating metamaterials and GCDWL structures into the plasmonic chip is complicated $[25,26]$.

Compared with complicated metamaterials and GCDWL structures, the graphene nanoribbon waveguide structure has obtained widespread attention and achievements because of its easy integration, ultranarrow bandwidth, high transmission, and relatively simple fabrication [27-29]. For instance, Wang et al. proposed tunable PIT effect based on Fabry-Perot resonance in a system by using two rectangle graphene cavities coupled with a graphene nanoribbon waveguide, which causes a relatively wide transmission window [30]. Noual et al. designed a compact graphene-based waveguide coupled to two rectangular cavity structures comprising graphene nanoribbons. In addition, the peak of the PIT window is less than 0.6 in this structure [31]. Zhao et al. reported that graphene nanoribbon systems comprise a graphene strip loaded with a complex structure of graphene stub and ring cavity, thereby obtaining a considerably sharp PIT window. However, only a single-band PIT effect is acquired in the system [32]. Therefore, a dynamic tunable multiband PIT effect realized by graphene nanoribbon waveguide structure in the $\mathrm{THz}$ band remains relatively challenging.

Inspired by this basic research, a dynamically tunable multiband PIT effect based on the graphene rectangle cavities coupled with a graphene nanoribbon waveguide is proposed by tuning the chemical potential of the graphene rectangle cavity. Two methods based on a different physical mechanism are used to realize a single-PIT effect; one is the bright and dark mode coupling through direct destructive interference, and the other is the cavity coupled with a graphene nanoribbon waveguide through 
indirect destructive interference. Moreover, dual- and triple-PIT effects are predicted in this ultracompact structure, whereas the corresponding group indexes are controlled from 143.2 to 108.6 and 161.4 to 115.8. The problems on the PIT window with low transmission, single band, and untunability in plasmonic waveguide devices applied to integration can be worked out particularly well, thereby demonstrating a new door for the dynamic tuning of slow light and dynamically tunable multichannel light storage devices.

\section{System Model Design And Theoretical Analysis}

Fig. 1 shows four types of models, which comprise remarkable graphene rectangle cavities and a graphene nanoribbon waveguide. The width of the graphene nanoribbon waveguide is only $10 \mathrm{~nm}$ in the PIT system because only the edge mode of the SPPs on the graphene nanoribbon is considered ${ }^{6}$. The edge modes are achieved by the incident light source placed in the $x$-direction of the system in Fig. 1. The incident light wave is restricted to travel forward in the waveguide when the incident light is injected and travels in the waveguide. SPPs are coupled into the graphene rectangle cavity as the SPP wave passes through the cavity-waveguide coupling region due to the near-field coupling. The graphene nanoribbon waveguide and rectangle cavities are placed on a $200 \mathrm{~nm}$ thick sapphire substrate to avoid substrate loss in the mid-infrared band. Only propagation loss as low as $1.9 \mathrm{~dB} / \mathrm{cm}$ at $5.18 \mu \mathrm{m}$ are caused in silicon-on-sapphire (SOS) waveguide [33]. $\mathrm{Al}_{2} \mathrm{O}_{3}$ is selected as the substrate material mainly because of the low loss of SPPs that traveled on the sapphire in the THz band [33]. The length of the rectangle cavity is $w_{\mathrm{a}}=w_{\mathrm{b}}=w_{\mathrm{c}}=w_{\mathrm{d}}=140 \mathrm{~nm}$, and the width of the rectangle cavity is $d_{\mathrm{a}}=d_{\mathrm{b}}=d_{\mathrm{c}}$ $=d_{\mathrm{d}}=20 \mathrm{~nm}$ [17]. In addition, the coupling distance between the rectangle cavities and the graphene nanoribbon waveguide is $l_{\mathrm{a} 2}=15 \mathrm{~nm}, l_{\mathrm{b} 1}=l_{\mathrm{b} 2}=15 \mathrm{~nm}, l_{\mathrm{c} 1}=l_{\mathrm{c} 2}=l_{\mathrm{c} 3}=15 \mathrm{~nm}, l_{\mathrm{d} 1}=l_{\mathrm{d} 3}=15 \mathrm{~nm}$, and that between the bright and dark modes is $l_{\mathrm{a} 1}=l_{\mathrm{d} 2}=l_{\mathrm{d} 4}=20 \mathrm{~nm}$ [17]. The simulation results reveal that the chemical potential of the graphene nanoribbon waveguide is constantly maintained at $0.40 \mathrm{eV}$, and the chemical potential of the graphene rectangle cavity is tuned by changing the applied voltages. 
(a)

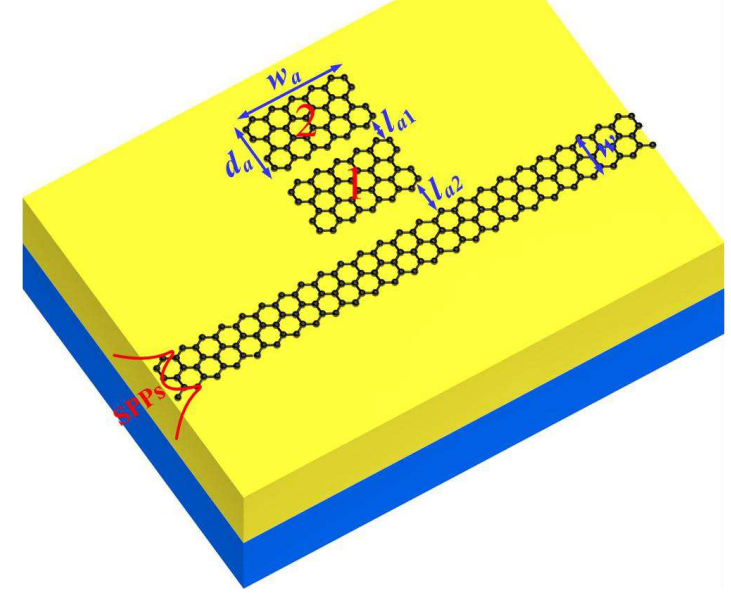

(c)

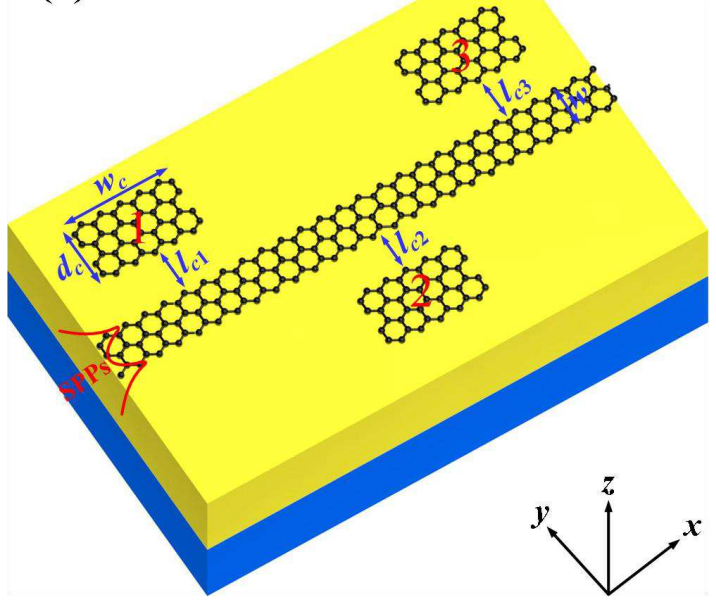

(b)

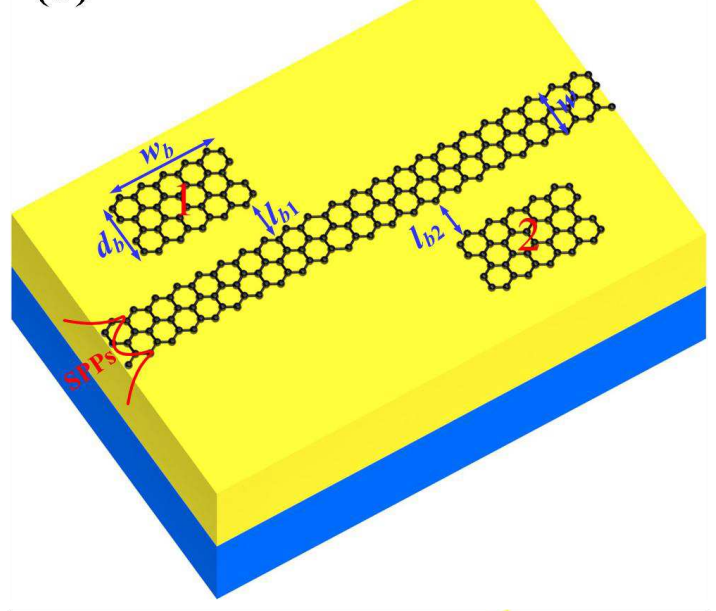

(d)

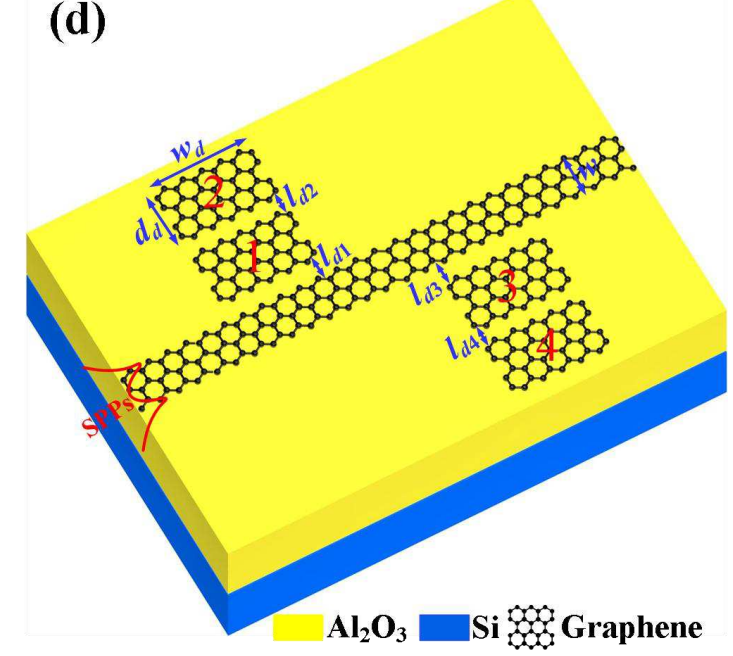

Fig. 1 Schematic of the proposed two-cavity-coupled system a-b, three-cavity-coupled system $\mathbf{c}$, and four-cavity-coupled system $\mathbf{d}$

The conductivity of the single-layer graphene $\sigma_{\mathrm{g}}$ can be expressed by Kubo formula, which represents the sum of the interband and intraband contributions: $\sigma_{\mathrm{g}}(\omega)=\sigma_{\text {inter }}(\omega)+\sigma_{\text {intra }}(\omega)$. The specific expression is as follows [34]:

$$
\sigma_{g}(\omega)=-i \frac{e^{2} k_{B} T}{\pi \hbar^{2}\left(\omega+i \tau^{-1}\right)}\left[\frac{E_{F}}{k_{B} T}+2 \ln \left(\exp \left(-\frac{E_{F}}{k_{B} T}\right)+1\right)\right]-i \frac{e^{2}}{4 \pi \hbar^{2}} \ln \left[\frac{2\left|E_{F}\right|-\hbar\left(\omega+i \tau^{-1}\right)}{\left.2 \mid E_{F}+\hbar \phi+i \tau^{-1}\right)}\right],
$$

where $i, T, k_{B}, e, \tau$, and $\hbar$ represent the imaginary unit, the temperature, the Boltzmann constant, the electron charge, the carrier relaxation time, and the Planck constant, respectively. $\omega$ is the angular frequency, $E_{\mathrm{F}}$ is the chemical potential of the grapheme, and the main contributions of $\sigma_{\mathrm{g}}$ are from the intraband electron-photon scattering, whereas those of $\sigma_{\mathrm{g}}$ can be negligible from the direct transitions of the interband electron. Therefore, the conductivity $\sigma_{\mathrm{g}}$ of monolayer graphene can be obtained as a Drude-like expression after simplification $[34,35]$.

$$
\sigma_{g}=\frac{i e^{2} E_{F}}{\pi \hbar^{2}\left(\omega+i \tau^{-1}\right)}
$$

The carrier relaxation time $\tau=\mu_{0} E_{\mathrm{F}} /\left(e v f^{2}\right)$, which is determined by the chemical potential of graphene $E_{\mathrm{F}}$, the carrier mobility of graphene $\mu_{0}$, and the Fermi velocity $v_{f}\left(\approx 10^{6} \mathrm{~m} / \mathrm{s}\right)$. The propagation 
constant $\beta$ of the monolayer graphene can be obtained using electromagnetic field boundary conditions and Maxwell equations to analyze the graphene properties effectively [17].

$$
\beta=k_{0} \sqrt{1-\left(\frac{2}{\eta_{0} \sigma_{\mathrm{g}}}\right)^{2}},
$$

where $k_{0}$ and $\eta_{0}$ are the wavenumber and the impedance in the free space, respectively. Therefore, the effective refractive index is expressed by $n_{\text {eff }}=\beta / k_{0}$, and the intrinsic quality factor is $Q_{\text {int }}=\operatorname{Re}\left(n_{\text {eff }}\right) /$ $\operatorname{Im}\left(n_{\text {eff }}\right)$. [35] Figure 2 shows the relationship of variation between the intrinsic quality factor and wavelength at various chemical potentials of the graphene. The intrinsic quality factor decreases with the respective increase in wavelength and the chemical potential of the graphene for the same wavelength, as shown in Fig. 2.

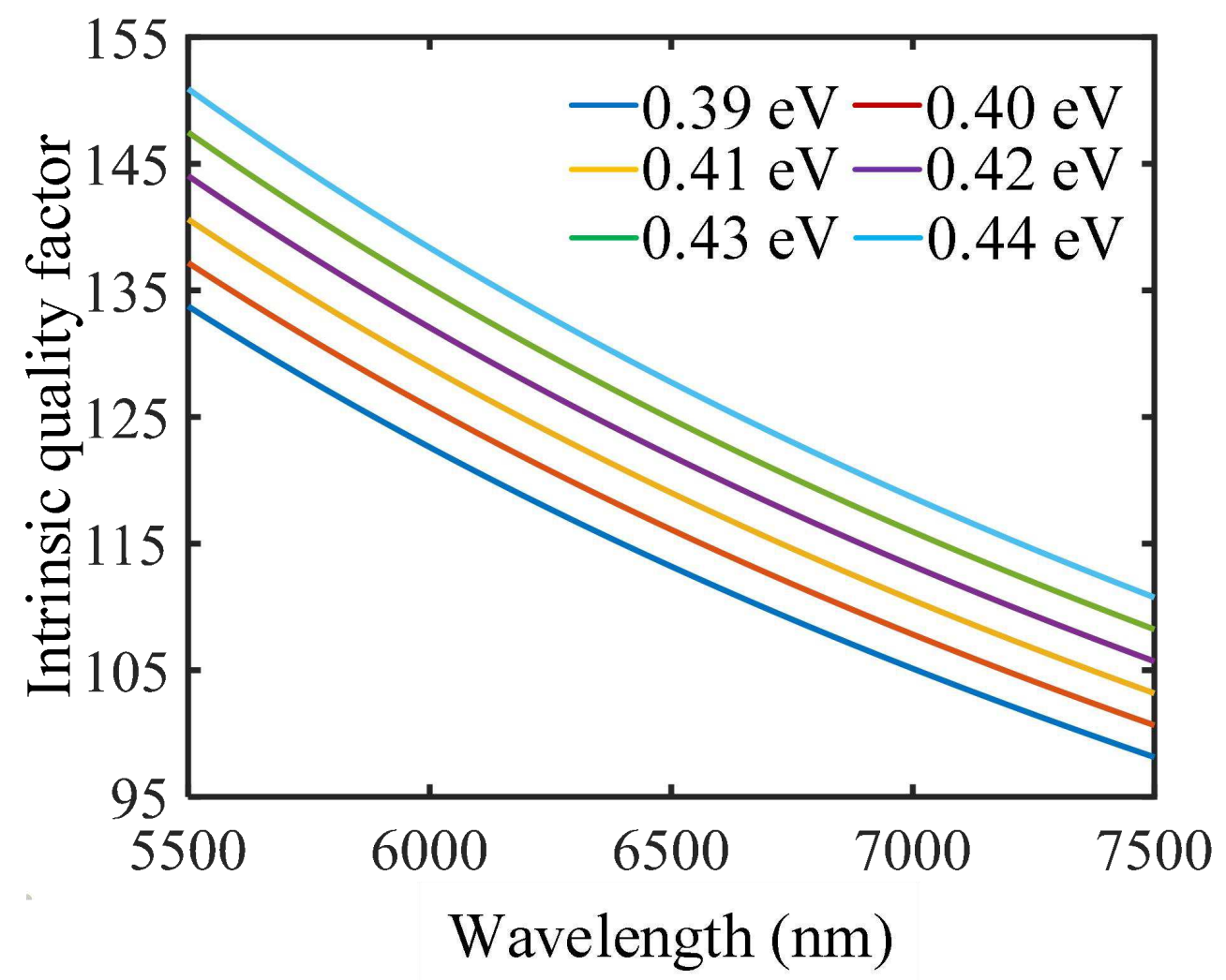

Fig. 2 Relationship between the intrinsic quality factor and the wavelength for various chemical potentials of the graphene $E_{F}=0.39,0.40,0.41,0.42,0.43$, and $0.44 \mathrm{eV}$

The chemical potential of the graphene rectangle cavity is tuned from $0.39 \mathrm{eV}$ to $0.44 \mathrm{eV}$ to obtain dynamically tunable and multiband PIT effect and multichannel slow light. The resonant wavelength of the graphene rectangle cavity can be expressed as follows: [17]

$$
\lambda=2 \operatorname{Re}\left(n_{\text {eff }}\right) W /(m-\varphi / \pi),
$$

where $W$ is the coupling length between the graphene nanoribbon waveguide and the rectangle cavities, $\varphi$ is the phase shift due to the reflection of the incident light in the cavity, and $m$ is an integer. 


\section{Simulation Results And Discussion Of Single-PIT Effect In Bright And Dark Mode Coupling Systems}

Figure 1(a) shows that the two-cavity-coupled system comprises rectangle cavity 1, rectangle cavity 2 , and the graphene nanoribbon waveguide. The chemical potential of graphene rectangle cavities 1 and 2 is initially set as 0.40 and $0.42 \mathrm{eV}$, respectively. The bright mode can be directly activated by the SPPs wave, whereas the dark mode can be indirectly excited by the bright mode. In addition, the light can be coupled back to the bright mode, which results in the higher coupling strength between rectangle cavity 1 and graphene nanoribbon waveguide than that between cavity 2 and the waveguide. Therefore, cavities 1 and 2 behave as bright and dark modes, respectively [36]. Notably, the graphene-based nanoribbon waveguide, the graphene rectangle cavity 1, and the graphene rectangle cavity 2 can serve as the bare element $|1\rangle$, the dipole-allowed element $|2\rangle$, and the metastable element $\mid 3>$, respectively [37]. Two optical pathways, namely, $|1>\rightarrow| 2>$ and $|1>\rightarrow| 2>\rightarrow|3>\rightarrow| 2>$, are observed in the proposed bright and dark mode coupling system. The bright cavity 1 and dark cavity 2 induce the transmission peaks because of the destructive interference with each other.

Figure 3 shows a schematic of the realizing principle according to the coupled mode theory (CMT) of the single-PIT effect in bright and dark mode coupling systems. The coupling mode equation is calculated, the single-PIT phenomena are analyzed, and the transmission and coupling loss of light wave are ignored. The dynamic equation of cavity mode amplitude $a_{i}(i=1,2)$ for the time-harmonic field $e^{-j \omega t}$ is as follows: [36]

$$
\begin{aligned}
& \frac{d a_{1}}{d t}=\left(j \omega_{0}-\kappa_{\mathrm{int}, 1}-\kappa_{\mathrm{w}}\right) a_{1}+\sqrt{\kappa_{\mathrm{w}}} S_{+, \mathrm{in}}^{(1)}-j \mu a_{2}, \\
& \frac{d a_{2}}{d t}=\left(j \omega_{0}-\kappa_{\mathrm{int}, 2}\right) a_{2}-j \mu a_{1},
\end{aligned}
$$

where $S_{p, \text { in }}^{(i)}$ and $S_{p, \text { out }}^{(i)}(i=1,2)$ represent the transmitted waveguide mode amplitude of the input and output light waves, respectively, and the subscript $p= \pm$ represents propagation directions of the positive and negative the light waves. $\omega_{0}$ is the intrinsic resonant frequency of the bright and dark modes, $\kappa_{\mathrm{w}, i}=1 / \tau_{\mathrm{w}, i}=\omega_{i} /\left(2 Q_{\mathrm{w}, i}\right)$ is the attenuation ratio on account of energy coupling to the graphene nanoribbon, $\kappa_{\mathrm{int}, i}=1 / \tau_{\mathrm{int}, i}=\omega_{i} /\left(2 Q_{\mathrm{int}, i}\right)$ is the attenuation ratio on account of the intrinsic quality loss in the $i$ th rectangle cavity, and $\mu=\omega_{0} /\left(2 Q_{\mathrm{c}}\right)$ is the coupling coefficient between the bright and dark mode. $Q_{\mathrm{w}}, Q_{\mathrm{int}, i}$, and $Q_{\mathrm{c}}$ are the waveguide coupling loss, the cavity quality factors related to the intrinsic loss, and the direct coupling between two rectangle cavities, respectively. finite difference time domain (FDTD) simulation reveals the following: $Q_{\mathrm{int}, 1}=113.2, Q_{\mathrm{int}, 2}=122.5, Q_{\mathrm{t}}=38.4, Q_{\mathrm{w}}=58.17$, and $Q_{\mathrm{c}}=$ 16.7 . 


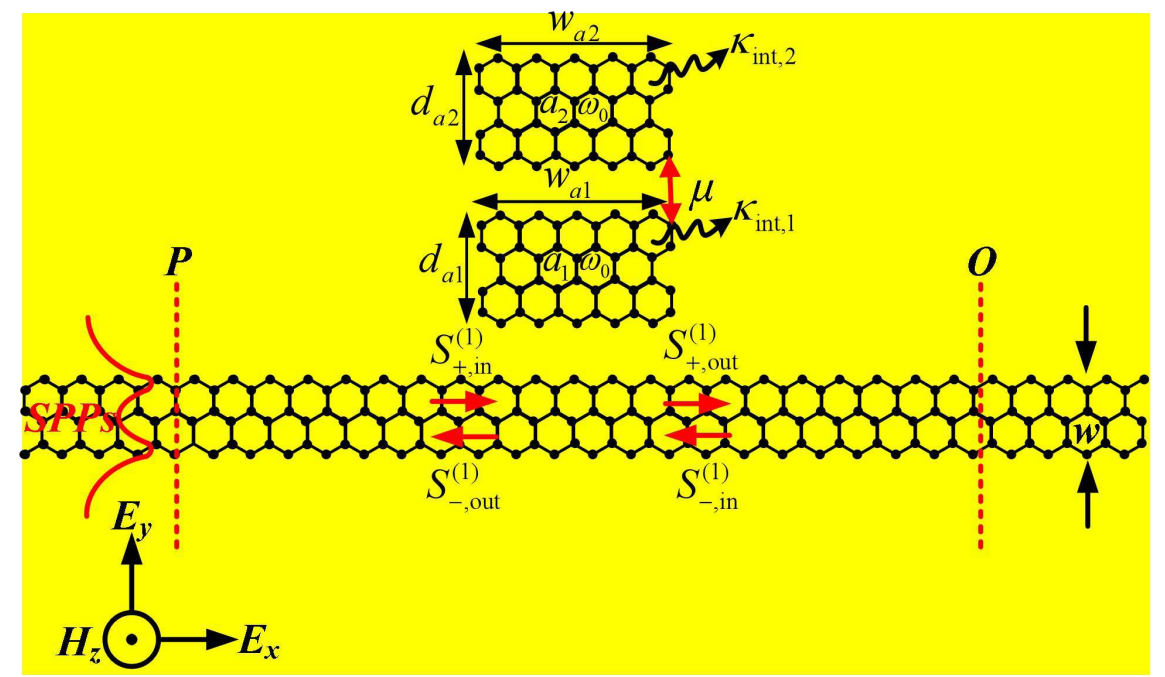

Fig. 3 Schematic showing the realization of the principle of the single-PIT effect in bright and dark mode coupling systems

The conservation of energy indicates that the output wave amplitude of each cavity can be expressed as follows:

$$
\begin{aligned}
& S_{+, \text {out }}^{(1)}=S_{+, \text {in }}^{(1)}-\sqrt{\kappa_{\mathrm{w}}} a_{1}, \\
& S_{-, \text {out }}^{(1)}=S_{-, \text {in }}^{(1)}-\sqrt{\kappa_{\mathrm{w}}} a_{1},
\end{aligned}
$$

The output spectral transmission of the system for the coupling of bright and dark mode systems is as follows:

$$
T_{1}=\left|t_{a}\right|^{2}=\left|\frac{S_{+, \text {out }}^{(1)}}{S_{+, \text {in }}^{(1)}}\right|^{2}=\left|1-\frac{\kappa_{\mathrm{w}}}{j\left(\omega-\omega_{0}\right)+\kappa_{\mathrm{int}, 1}+\kappa_{\mathrm{w}}+\frac{\mu^{2}}{j\left(\omega-\omega_{0}\right)+\kappa_{\mathrm{int}, 2}}}\right|^{2},
$$

where $t_{a}$ is the transmission coefficient of bright and dark mode coupling systems.

The transmission phase shift is $\theta(\omega)=\arg \left(t_{a}\right)$, and the group delay of this system is $\tau_{\mathrm{g}}=\partial \theta(\omega) / \partial \omega$. The slow light effect can be obtained by the group index $n_{\mathrm{g}}$, which is derived by the following: [22]

$$
n_{\mathrm{g}}=\frac{c}{v_{\mathrm{g}}}=\frac{c}{l} \tau_{\mathrm{g}}=\frac{c}{l} \cdot \frac{\partial \theta(\omega)}{\partial \omega},
$$

where $c, v_{\mathrm{g}}$, and $l$ are the vacuum velocity of incident light, the group velocity, and the coupling length of monolayer graphene nanoribbon waveguide system, respectively.

The transmission spectra of the single-PIT effect under various graphene chemical potentials in bright and dark mode coupling systems are shown in Figs. 4(a1)-(d1). The chemical potential of cavity 1 is constantly maintained as $E_{\mathrm{F} 1}=0.40 \mathrm{eV}$, while that of cavity 2 is set at different values $E_{\mathrm{F} 2}=0.39$, $0.40,0.41$, and $0.42 \mathrm{eV}$. The transmission peak has an evident blue shift as the chemical potential of cavity 2 increases, and that of the PIT effect becomes slightly high, as shown in Figs. 4(a1)-4(d1). The data of the FDTD and CMT calculation are highly consistent. Consequently, the single-PIT effect can 
be dynamically tuned in this structure by tuning the chemical potential of cavity 2 . Figs. 4(a1) and 4(d1) show that the transmission peaks of the PIT effect are $58 \%$ and $66 \%$ when the chemical potential of cavity 2 is 0.39 and $0.42 \mathrm{eV}$, respectively. Therefore, the high transmittance of the single-PIT effect is maintained when the chemical potential of the graphene rectangle cavity is tuned from $0.39 \mathrm{eV}$ to 0.42 $\mathrm{eV}$. This finding indicates that the dynamically tunable single-PIT effect can be obtained by adjusting the chemical potential of the graphene rectangle cavity 2 .
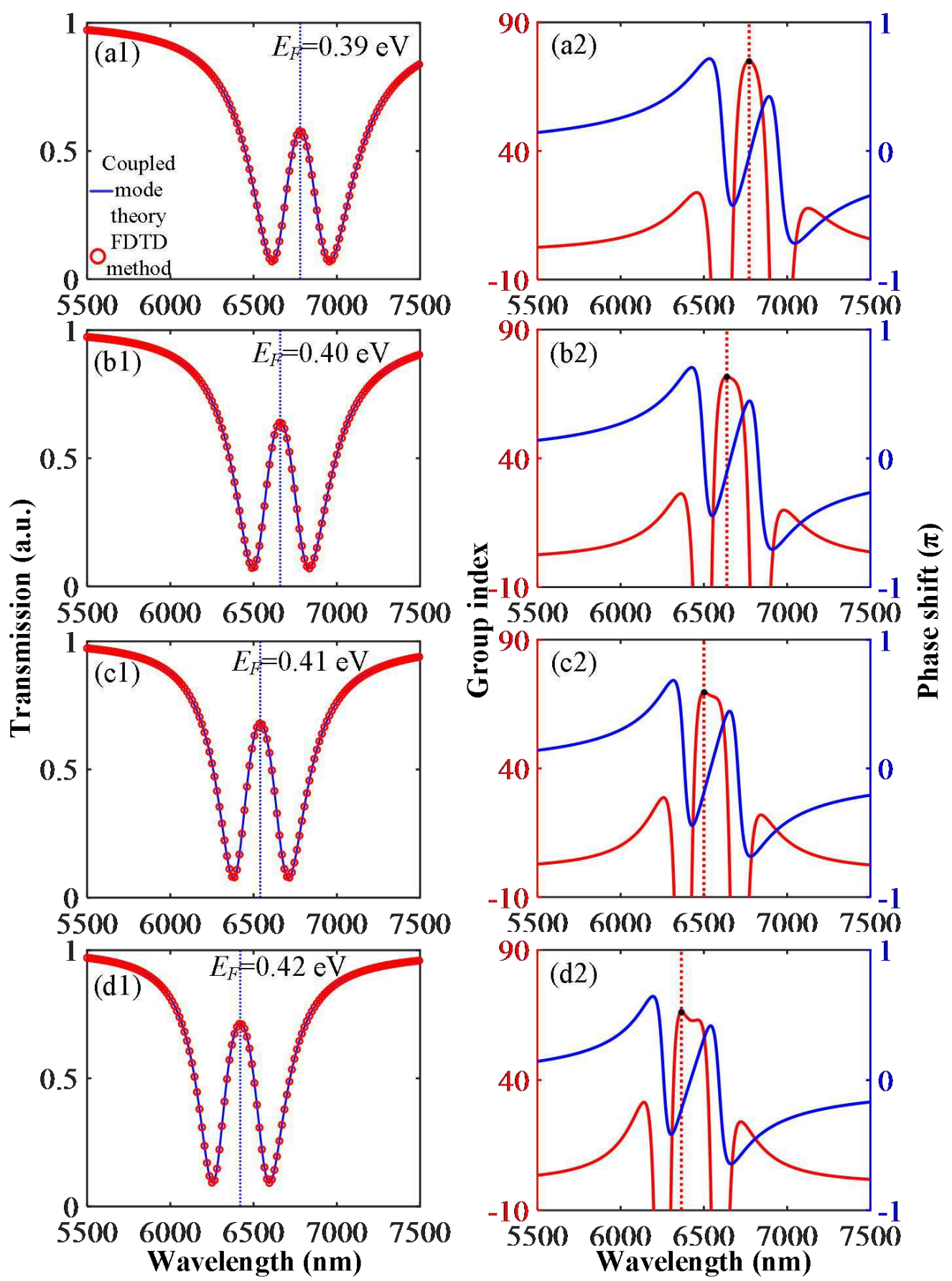

Fig. 4 Single-PIT effect simulation analysis of bright and dark mode coupling systems. (a1)-(d1) Transmission spectra of single-PIT effect. (a2)-(d2) Corresponding transmission phase shift responses and group index 
Figs. 4(a2)-(d2) show transmission phase shift responses and group index under various graphene chemical potentials (from $0.39 \mathrm{eV}$ to $0.42 \mathrm{eV}$ ) in bright and dark mode coupling systems. Figs. 4(a2)(d2) show that the phase shift is $2 m \pi$ at the peak wavelength because the dispersions are strong in the transparent wavelength. The group index at the peak wavelength and the transmission peak wavelength of the PIT effect decrease with the increase in graphene chemical potential of cavity 2 , as shown in Figs. 4(a2)-(d2). Meanwhile, the intensity of the PIT transparent peak slightly decreases as the group index increases. This trend may be due to the additional time spent by the signal light in both rectangle cavities, thereby resulting in increased light power loss by scattering due to the resonance in the cavities. The group indexes of the peak wavelength are 74.9, 71.7, 69.6, and 66.1 with the graphene chemical potential of cavity 2 of $0.39,0.40,0.41$, and $0.42 \mathrm{eV}$, respectively, as shown in the black dots of Figs. 4(a2)-(d2).

Fig. 5 shows that the magnetic field simulation of $\left|H_{z}\right|^{2}$ is depicted at the transmission peak wavelength to further understand the bright and dark mode coupling mechanism of this structure. This figure also reveals the magnetic field distribution at the peak wavelength of $6420 \mathrm{~nm}$ when the chemical potential of cavities 1 and 2 are set to 0.40 and $0.42 \mathrm{eV}$, respectively, and the corresponding transmission spectrum of the PIT effect is shown in Fig. 4(d1). The bright cavity is directly excited, whereas the dark cavity is activated by coupling with the bright one. Hence, only the dark cavity is efficiently activated, whereas the bright cavity is evidently suppressed at the transmission peak. Similar to the EIT effect in atom systems, canceling of the bright cavity is attributed to the destructive interference caused by two optical pathways from graphene nanoribbon waveguide to the graphene rectangle cavity.

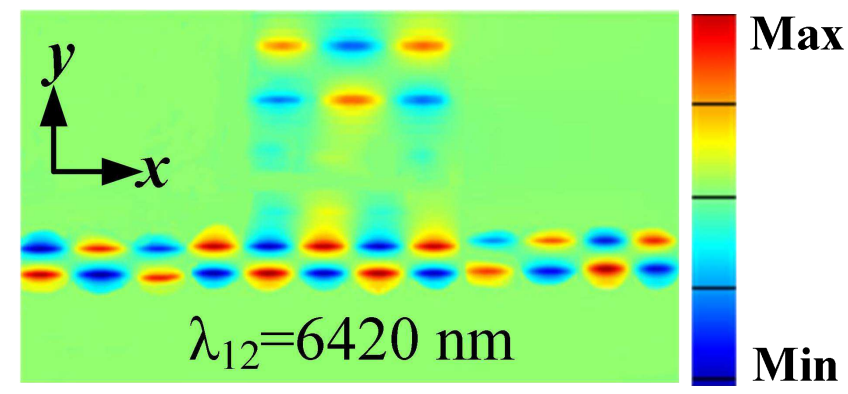

Fig. $5\left|H_{z}\right|^{2}$ magnetic field distributions of the single-PIT effect in bright and dark mode coupling systems at the transparent wavelength

\section{Simulation Results And Discussion Of Single-PIT Effect In Two Cavities Side-Coupled With Waveguide System}

Fig. 1(b) shows that the PIT system comprises rectangle cavities 1 and 2 side-coupled with a graphene nanoribbon waveguide. The chemical potentials of graphene rectangle cavities 1 and 2 are initially set to 0.40 and $0.44 \mathrm{eV}$, respectively. Fig. 6 reveals the analysis of the dynamic transmission features for two cavities side-coupled with a graphene nanoribbon waveguide system using a temporal CMT. The normalized amplitude $a_{i}$ for the time-harmonic field $e^{-j \omega t}$ of the $i$ th cavity $(i=1,2)$ for the time-harmonic field $e^{-j \omega t}$ is provided by the following: [16] 


$$
\begin{gathered}
\frac{d a_{1}}{d t}=\left(-j \omega_{1}-\kappa_{\mathrm{int}, 1}-\kappa_{\mathrm{w}, 1}\right) a_{1}+\sqrt{\kappa_{\mathrm{w}, 1}} S_{+, \mathrm{in}}^{(1)}+\sqrt{\kappa_{\mathrm{w}, 1}} S_{-, \mathrm{in}}^{(1)}, \\
\frac{d a_{2}}{d t}=\left(-j \omega_{2}-\kappa_{\mathrm{int}, 2}-\kappa_{\mathrm{w}, 2}\right) a_{2}+\sqrt{\kappa_{\mathrm{w}, 2}} S_{+, \text {in }}^{(2)}+\sqrt{\kappa_{\mathrm{w}, 2}} S_{-, \mathrm{in}}^{(2)},
\end{gathered}
$$

where $\omega_{i}(i=1,2)$ are the intrinsic resonant frequencies of the two cavities, $\kappa_{\mathrm{w}, i}(i=1,2)$ is the attenuation ratio due to energy coupling into the plasmonic waveguide, and $\kappa_{\mathrm{int}, i}(i=1,2)$ is the attenuation ratio of the $i$ th rectangle cavity; $Q_{\mathrm{w} 1}=23.1, Q_{\mathrm{w} 2}=22.5, Q_{\mathrm{int}, 1}=127.7, Q_{\mathrm{int}, 2}=132.2$, and $Q_{\mathrm{t}}=$ 19.1 .

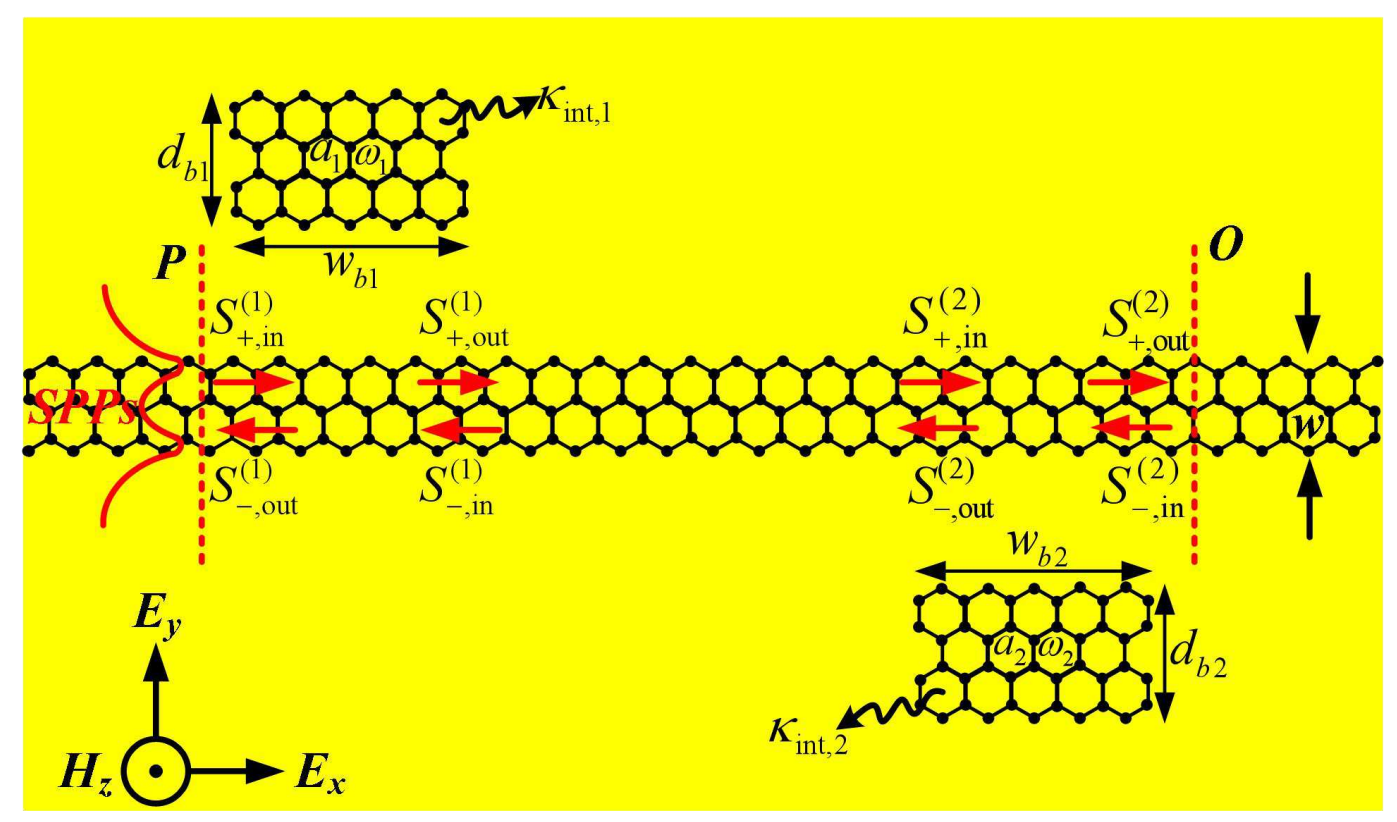

Fig. 6 Schematic showing the realization of the principle of the single-PIT effect in two cavities side-coupled with a graphene nanoribbon waveguide

The current study assumes the presence of only the light of the positive $x$ direction $\left(S_{- \text {,in }}^{(2)}=0\right)$, and the transmission and reflection coefficients of a single cavity side-coupled with a graphene nanoribbon waveguide system are expressed as follows:

$$
\begin{gathered}
t_{i}(\omega)=\frac{j\left(\omega_{i}-\omega\right)+\kappa_{\mathrm{int}, i}}{j\left(\omega_{i}-\omega\right)+\kappa_{\mathrm{int}, i}+\kappa_{\mathrm{w}, i}}, \\
r_{i}(\omega)=-\frac{\kappa_{\mathrm{w}, i}}{j\left(\omega_{i}-\omega\right)+\kappa_{\mathrm{int}, i}+\kappa_{\mathrm{w}, i}},
\end{gathered}
$$

The output transmitted efficiency for the two graphene rectangle cavities side-coupled with a graphene nanoribbon waveguide system can be expressed as follows [16]

$$
T_{2}=\left|t_{b}\right|^{2}=\left|\frac{S_{+, \mathrm{out}}^{(2)}}{S_{+, \text {in }}^{(1)}}\right|^{2}=\left|\frac{t_{1}(\omega) t_{2}(\omega)}{1-r_{1}(\omega) r_{2}(\omega) e^{j \phi}}\right|^{2},
$$

where $t_{b}$ is the transmission coefficient of two graphene rectangle cavities side-coupled with a graphene nanoribbon waveguide system, and $\phi$ is the phase shift between two rectangle cavities.

Fig. 7 shows the results of the transmission spectra, corresponding transmission phase shift 
responses, and group index under different graphene chemical potentials (from $0.41 \mathrm{eV}$ to $0.44 \mathrm{eV}$ ). The resonant wavelength of rectangle cavity 2 is blueshifted as the graphene chemical potential of cavity 2 increases, and the wavelength detuning between the two cavities increases. The PIT peak wavelength blueshifts because of the reduction in effective refractive index. The transmission spectrum shows a PIT peak because of the strong coupling between two cavities and the phase shift value of $2 \mathrm{~m} \pi$ (where $\mathrm{m}$ is an integer). Figs. 7(a2)-(d2) show that the transmission phase shift is $0 \pi$ at the peak wavelength due to the Fabry-Perot resonance. Figs. 7(a1)-(d1) reveal the gradual increase in transparent peak as the wavelength detuning increases, indicating an increase in coherent interference between the two rectangle cavities. Fig. 7(a1) proves that the bandwidth is ultranarrow when the graphene chemical potential of cavity 2 is $0.41 \mathrm{eV}$, and a low PIT peak is obtained. Fig. 7(d1) shows that the bandwidth reaches a maximum when the graphene chemical potential of cavity 2 is increased to $0.44 \mathrm{eV}$, and the peak value of PIT reaches $62 \%$. This result indicates that strong destructive interference is achieved between the two rectangle cavities.

The maximum delay of this PIT system is inversely proportional to the bandwidth of transmission because of the principle of the delay bandwidth product, resulting in a tradeoff between the group index and wavelength detuning. Thus, the group index decreases as the wavelength detuning increases at the peak wavelength, as indicated in Figs. 7(a2)-(d2). Meanwhile, the intensity of the PIT transparent peak decreases as the group index increases. This phenomenon is due to the additional time spent by the signal light in both rectangle cavities, resulting in increased light power loss by resonance in the cavities. The group indexes of the peak wavelength are 79.2, 53.2, 37.4, and 28.3 with the graphene chemical potentials of cavity 2 of $0.41,0.42,0.43$, and $0.44 \mathrm{eV}$, respectively, as shown in the black dots of Figs. 7(a2)-(d2). 

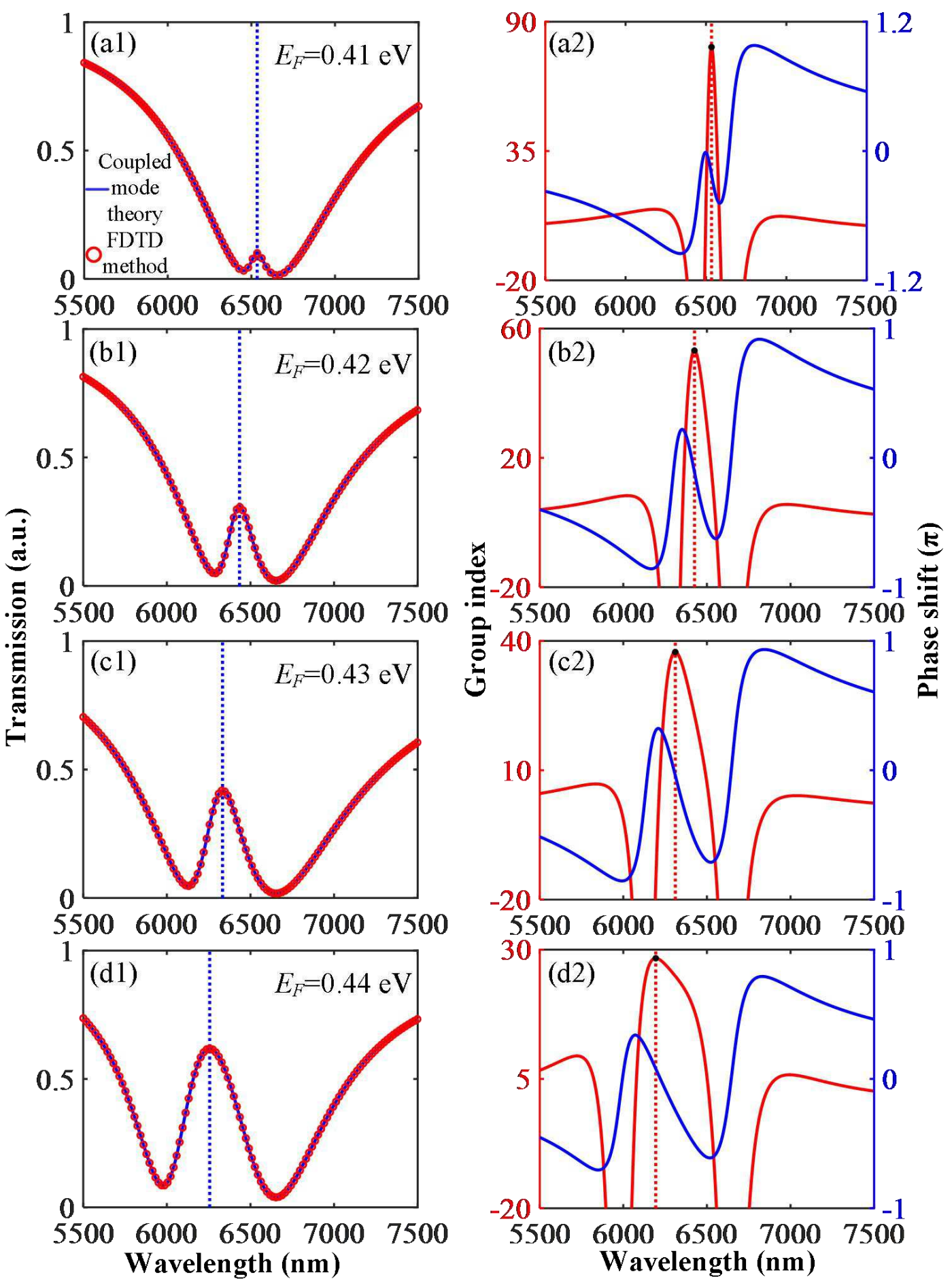

Fig. 7 Single-PIT effect simulation analysis of two cavities side-coupled with a graphene nanoribbon waveguide system. a1-d1 Transmission spectrum of the single-PIT effect. a2-d2 Corresponding transmission phase shift responses and group index

Figs. 8(a)-(c) show that the magnetic field simulation of $\left|H_{z}\right|^{2}$ is depicted at the transmission peak and the two dip wavelengths to further understand the coupling mechanism of this structure. Fig. 8(a) demonstrates the magnetic field distribution at the resonance wavelength of cavity 1 at $6650 \mathrm{~nm}$ when the chemical potentials of cavities 1 and 2 are set to 0.40 and $0.44 \mathrm{eV}$, respectively. Fig. $8(\mathrm{~b})$ shows the magnetic field distribution at the peak wavelength of the PIT effect at $6256 \mathrm{~nm}$, and the corresponding transmission spectrum of the PIT effect is presented in Fig. 7(d1). Fig. 8(c) shows the magnetic field 
distribution at the resonant wavelength of cavity 2 at $5983 \mathrm{~nm}$. The results show that Fabry-Perot resonance exists between the two rectangle cavities.

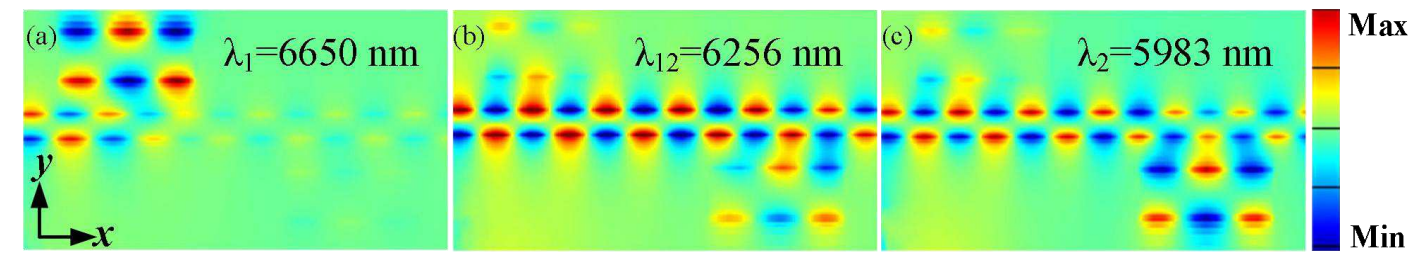

Fig. $8\left|H_{z}\right|^{2}$ magnetic field distributions of the single-PIT effect in two cavities side-coupled with a graphene nanoribbon waveguide system at the resonance wavelength

Table I shows the maximum transmission and group index of the single-PIT system under different chemical potentials of graphene rectangle cavities at the peak wavelength of PIT. The maximum transmission of the single-PIT effect of bright and dark mode coupling systems is tuned from $58 \%$ to $71 \%$ due to the changing chemical potential of the graphene rectangle cavity 2 from 0.39 $\mathrm{eV}$ to $0.42 \mathrm{eV}$, and the group index is controlled between 74.9 and 66.1. This condition indicates that a fine-tuning method of the group index can be obtained in this PIT system. Alternatively, the maximum transmission of the single-PIT effect of two cavities side-coupled with a graphene nanoribbon waveguide system is tuned from $10 \%$ to $62 \%$ due to the changing chemical potential of the graphene rectangle cavity 2 from $0.41 \mathrm{eV}$ to $0.44 \mathrm{eV}$, and the group index is controlled between 79.2 and 28.3. This condition indicates that the group index is tuned in a large tuning range by dynamically tuning the chemical potential of the graphene rectangle cavity. These results can be effectively applied to dynamically tunable light storage and slow light devices.

Table I Maximum transmission and group index of the single-PIT system under different chemical potentials of graphene rectangle cavities at the peak wavelength of PIT

\begin{tabular}{cccc}
\hline System model & $\begin{array}{c}\text { Chemical potentials of } \\
\text { graphene rectangle cavities }\end{array}$ & $\begin{array}{c}\text { Maximum } \\
\text { transmission } \\
\text { of PIT peak }\end{array}$ & $\begin{array}{c}\text { Maximum } \\
\text { group index of } \\
\text { PIT system }\end{array}$ \\
\hline \multirow{2}{*}{$\begin{array}{c}E_{F 1}=0.40 \mathrm{eV}, E_{F 2}=0.39 \mathrm{eV} \\
\text { dargle-PIT effect of bright and }\end{array}$} & $E_{F 1}=0.40 \mathrm{eV}, E_{F 2}=0.40 \mathrm{eV}$ & $64 \%$ & 74.9 \\
& $E_{F 1}=0.40 \mathrm{eV}, E_{F 2}=0.41 \mathrm{eV}$ & $67 \%$ & 71.7 \\
& $E_{F 1}=0.40 \mathrm{eV}, E_{F 2}=0.42 \mathrm{eV}$ & $71 \%$ & 69.6 \\
Single-PIT effect of two cavities & $E_{F 1}=0.40 \mathrm{eV}, E_{F 2}=0.41 \mathrm{eV}$ & $10 \%$ & 79.2 \\
side-coupled with a waveguide & $E_{F 1}=0.40 \mathrm{eV}, E_{F 2}=0.42 \mathrm{eV}$ & $31 \%$ & 53.2 \\
& $E_{F 1}=0.40 \mathrm{eV}, E_{F 2}=0.43 \mathrm{eV}$ & $41 \%$ & 37.4 \\
system & $E_{F 1}=0.40 \mathrm{eV}, E_{F 2}=0.44 \mathrm{eV}$ & $62 \%$ & 28.3 \\
\hline
\end{tabular}

\section{Simulation Results And Discussion Of Dual-PIT Effect In Triple Cavities Side-Coupled With A Graphene Nanoribbon Waveguide System}

Fig. 1(c) shows that the dual PIT system comprises rectangle cavity 1 , cavity 2 , cavity 3 , and a graphene nanoribbon waveguide. The graphene rectangle cavity 1, cavity 2, and cavity 3 have an initial 
chemical potential of $0.42,0.43$, and $0.44 \mathrm{eV}$, respectively. Fig. 9 shows that the dynamic transmission features for triple graphene rectangle cavities side-coupled with a graphene nanoribbon waveguide system are analyzed by a temporal CMT. The normalized amplitude $a_{i}$ for the time-harmonic field $e^{-j \omega t}$ of the $i$ th cavity $(i=1,2,3)$ are analyzed by the following:

$$
\begin{aligned}
& \frac{d a_{1}}{d t}=\left(j \omega_{1}-\kappa_{\mathrm{int}, 1}-\kappa_{\mathrm{w}, 1}\right) a_{1}+\sqrt{\kappa_{\mathrm{w}, 1}} S_{+, \mathrm{in}}^{(1)}+\sqrt{\kappa_{\mathrm{w}, 1}} S_{-, \mathrm{in}}^{(1)}, \\
& \frac{d a_{2}}{d t}=\left(j \omega_{2}-\kappa_{\mathrm{int}, 2}-\kappa_{\mathrm{w}, 2}\right) a_{2}+\sqrt{\kappa_{\mathrm{w}, 2}} S_{+, \mathrm{in}}^{(2)}+\sqrt{\kappa_{\mathrm{w}, 2}} S_{-, \mathrm{in}}^{(2)}, \\
& \frac{d a_{3}}{d t}=\left(j \omega_{3}-\kappa_{\mathrm{int}, 3}-\kappa_{\mathrm{w}, 3}\right) a_{3}+\sqrt{\kappa_{\mathrm{w}, 3}} S_{+, \mathrm{in}}^{(3)}+\sqrt{\kappa_{\mathrm{w}, 3}} S_{-, \mathrm{in}}^{(3)},
\end{aligned}
$$

where $\omega_{i}(i=1,2,3)$ represents the intrinsic resonant frequencies of the three cavities, $\kappa_{\mathrm{int}, i}(i=1,2,3)$ is the attenuation ratio of the $i$ th rectangle cavity, and $\kappa_{\mathrm{w}, i}(i=1,2,3)$ is the attenuation ratio due to energy coupling into the graphene nanoribbon waveguide.

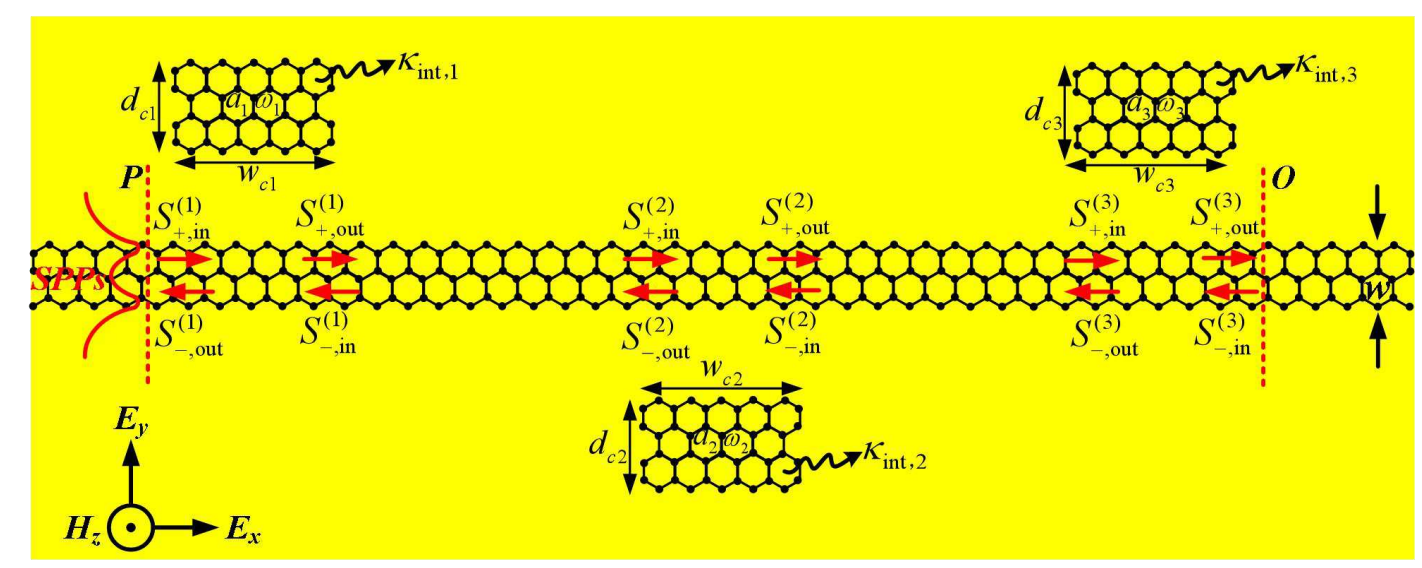

Fig. 9 Schematic showing the realization of the principle of the dual-PIT effect in triple cavities side-coupled with a waveguide system

The conservation of energy indicates that the output wave amplitude of each rectangle cavity can be analyzed $(i=1,2)$, as follows:

$$
\begin{aligned}
& S_{+, \text {out }}^{(i)}=S_{+, \text {in }}^{(i+1)} e^{j \phi_{i} / 2}, \\
& S_{-, \text {out }}^{(i+1)}=S_{-, \text {in }}^{(i)} e^{j \phi_{i} / 2},
\end{aligned}
$$

where $\phi_{1}$ is the transmission phase shift between cavities 1 and 2 , and $\phi_{2}$ is the transmission phase shift between cavities 2 and $3\left(\phi_{1}=\phi_{2}=\phi\right)$.

The output transmitted efficiency for triple graphene rectangle cavities side-coupled with a graphene nanoribbon waveguide system can be analyzed as follows:

$$
T_{3}=\left|t_{c}\right|^{2}=\left|\frac{S_{+, \mathrm{out}}^{(3)}}{S_{+, \mathrm{in}}^{2(1)}}\right|^{2}=\left|\frac{\alpha_{1} \alpha_{2} \alpha_{3}}{\left(\alpha_{1}+\kappa_{\mathrm{w}, 1}\right)\left(\alpha_{3}+\kappa_{\mathrm{w}, 3}\right) \alpha_{2} e^{i \phi}-\kappa_{\mathrm{w}, 1} \kappa_{\mathrm{w}, 3} \alpha_{2} e^{-j \phi}+\left(\alpha_{1}+\kappa_{\mathrm{w}, 1}\right)\left(\kappa_{\mathrm{w}, 2} \alpha_{3} e^{i \phi}+\kappa_{\mathrm{w}, 1} K_{\mathrm{w}, 3} e^{i \phi}-1\right)-\kappa_{\mathrm{w}, \mathrm{K}, K_{\mathrm{w}, 2}} \alpha_{3}+\kappa_{\mathrm{w}, 1} K_{\mathrm{w}, 2} \kappa_{\mathrm{w}, 3}\left(e^{-j \phi}-1\right)}\right|^{2},
$$

where $\alpha_{1}=j\left(\omega-\omega_{1}\right)+\kappa_{\mathrm{int}, 1}, \alpha_{2}=j\left(\omega-\omega_{2}\right)+\kappa_{\mathrm{int}, 2,}, \alpha_{3}=j\left(\omega-\omega_{3}\right)+\kappa_{\mathrm{int}, 3} ; t_{c}$ is the transmission coefficient of triple graphene rectangle cavities side-coupled with a graphene nanoribbon waveguide system. 
FDTD simulations revealed that the corresponding resonant wavelengths of rectangle cavities 1, 2, and 3 are $[6815,6625$, and $6435 \mathrm{~nm}],[6650,6460$, and $6270 \mathrm{~nm}],[6483,6293$, and $6103 \mathrm{~nm}]$, and [6324, 6134, and $5944 \mathrm{~nm}]$ when the chemical potentials of rectangle cavities 1,2 , and 3 are [0.39, 0.40, and $0.41 \mathrm{eV}],[0.40,0.41$, and $0.42 \mathrm{eV}],[0.41,0.42$, and $0.43 \mathrm{eV}]$, and [0.42, 0.43, and $0.44 \mathrm{eV}]$. The corresponding intrinsic quality factors presented in Fig. 2 are as follows: $Q_{\text {int, } 1}=108.2,113.4$, $120.1,125.2 ; Q_{\text {int, }}=113.5,120.1,126.1,132.3 ; Q_{\text {int }, 3}=120.1,126.5,132.9,139.7$. The total quality factors are caused by the following two PIT transparent peaks: $Q_{\mathrm{t}, 1}=90.2,88.5 ; Q_{\mathrm{t}, 2}=80.4,77.6 ; Q_{\mathrm{t}, 3}=$ $72.7,70.3 ; Q_{\mathrm{t}, 4}=65.6,63.4$. Therefore, the corresponding coupling quality factors of rectangle cavities 1,2 , and 3 can be obtained in accordance with $Q_{\mathrm{w}}=Q_{\mathrm{int}} Q_{\mathrm{t}} /\left(Q_{\mathrm{int}}-Q_{\mathrm{t}}\right)$ as $Q_{\mathrm{w}, 1}=540.1,272.8,184.2$, 137.2; $Q_{\mathrm{w}, 2}=432.2,223.1,159.8,130.3 ; Q_{\mathrm{w}, 3}=329.2,197.1,149.5,116.3$, respectively.

Fig. 10(d1) shows that A, B, and C are the corresponding transmission dips when the chemical potentials of rectangle cavities 1,2 , and 3 are $0.42,0.43$, and $0.44 \mathrm{eV}$, respectively. Peak I is the PIT transparent peak, which is caused by the light resonance in cavities 2 and 3 . The amplitude of the input wave in cavity 1 is equal to that in cavity 2 because the light is not coupled into cavity 1 . Therefore, $S_{+, \text {in }}^{(1)}=S_{+, \text {in }}^{(2)}$, and $T_{3}^{\prime}=\left|\frac{S_{+, \text {out }}^{(3)}}{S_{+, \text {in }}^{(2)}}\right|^{2}$. Similarly, peak II, which is caused by the resonance of the light in cavities 1 and 2, is the PIT transparent peak. The amplitude of the output wave in cavity 2 is equal to that in cavity 3 because the light is not coupled into cavity 3 . Herein, $S_{+, \text {out }}^{(2)}=S_{+, \text {out }}^{(3)}$, and $T_{3}^{\prime \prime}=\left|\frac{S_{+, \text {out }}^{(2)}}{S_{+, \text {in }}^{(1)}}\right|^{2}$.

Figs. 10(a1)-(d1) show the normalized transmission intensity spectra of the dual-PIT effect under various chemical potentials of graphene rectangle cavities. The resonant wavelengths of cavities 1,2 , and 3 show a blue shift, and the two PIT transmission peaks increase with the chemical potentials of graphene rectangle cavities, as shown in Figs. 10(a1)-(d1). The transmission peaks I and II of the dualPIT effect are the minimum values of only $21 \%$ and $18 \%$, respectively, when the chemical potentials of graphene rectangle cavities 1,2 , and 3 are $0.39,0.40$, and $0.41 \mathrm{eV}$, respectively, as shown in Fig. 10(a1). However, the values of the transmission peaks I and II can reach $74 \%$ and $71 \%$, respectively, when the chemical potentials of graphene rectangle cavities 1,2 , and 3 are $0.42,0.43$, and $0.44 \mathrm{eV}$, respectively, as shown in Fig. 10(d1). These results indicate that the dual-PIT effect can be effectively tuned by adjusting the chemical potential of the graphene rectangle cavity. 

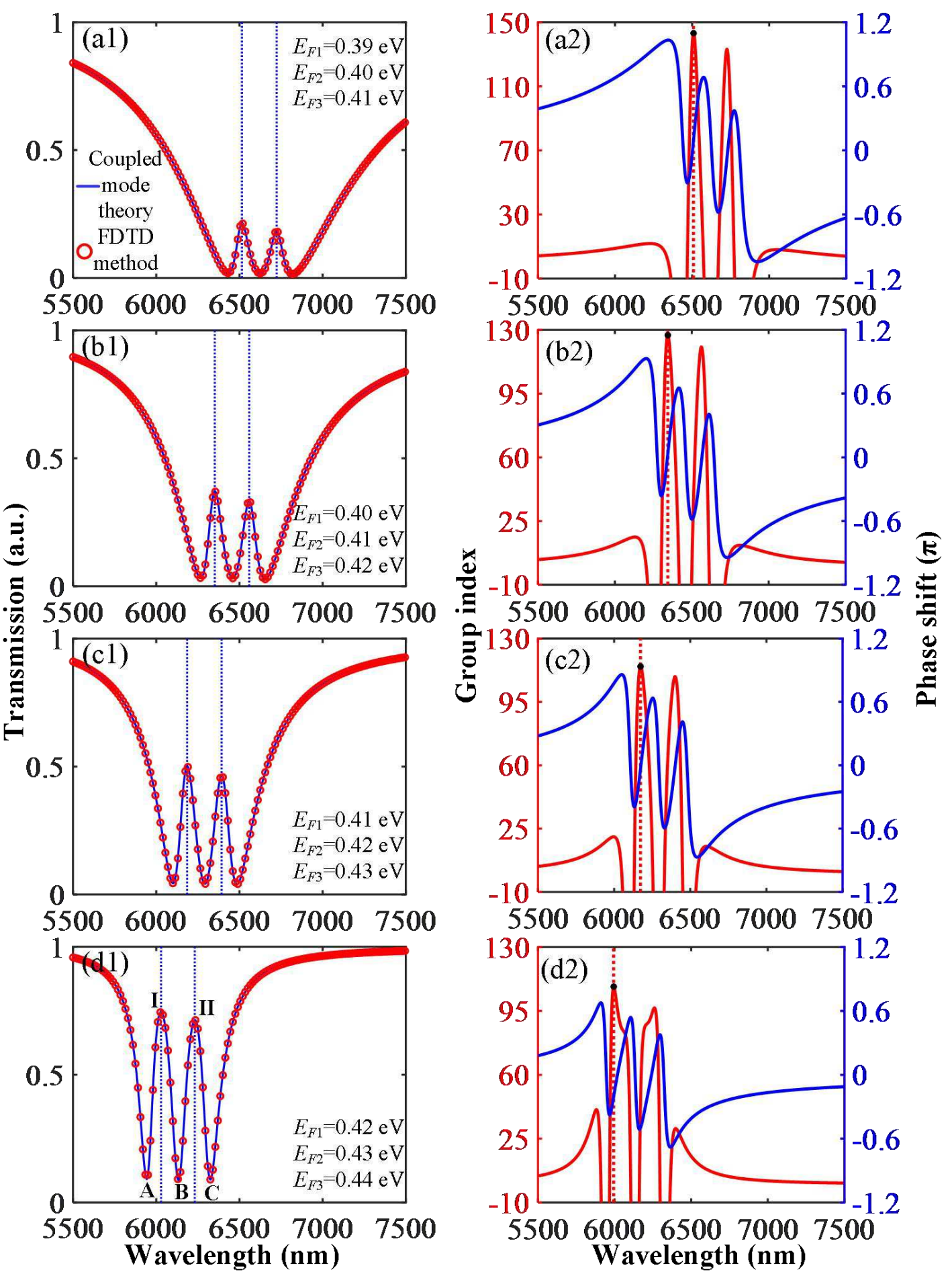

Fig. 10 Dual-PIT effect simulation analysis of triple cavities side-coupled with a graphene nanoribbon waveguide system. a1-d1 Transmission spectra of the dual-PIT effect. a2-d2 Corresponding transmission phase shift responses and group index

Figs. 10(a2)-(d2) show the transmission spectrum phase shift and group index of dual-PIT effect with the change in the chemical potential of graphene rectangle cavity. The results reveal the aggressive phase changes of the dual-PIT windows. The strong destructive interference near the peak of the transparency in the monolayer graphene-PIT structure results in a drastic dispersion. Hence, this phenomenon can induce a phase mutation, and the round-trip phase difference between the two cavities in the PIT system is an integer multiple of $2 \pi$. The transmission phase shifts of the dual-PIT effect 
caused by the light resonance in cavities 1 and 2 as well as cavities 2 and 3 are $0 \pi$. The dispersions are normal in the three cavity systems. Then, the phase dispersion results in a change in the group index. The group index of the dual-PIT system increases with the decrease in the chemical potential of the graphene rectangle cavity, and the two transmission peaks decrease, as shown in Figs. 10(a2)-(d2). This trend is due to the additional time spent by the signal light in resonance between cavities 1 and 2 as well as that between cavities 2 and 3, resulting in increased optical power loss among the three rectangle cavities. Therefore, the output light intensity of the dual-PIT system decreases with the graphene chemical potential. Moreover, a large group index is always maintained at the peak wavelength of the dual-PIT system. The corresponding maximum group indexes are 143.2, 127.3, 116.2, and 108.6, as shown in the black dots of Figs. 10(a2)-(d2). These results can be applied to dynamically tunable two-channel optical filters, optical modulators [38], and slow light devices because of high transmission and ultranarrow bandwidth [12].

The field distributions of $\left|H_{z}\right|^{2}$ at the two peak wavelengths are depicted by simulation at the transmission peak wavelengths, as shown in Figs. 11(a) and (b) to further understand the dual-PIT effect mechanism of this structure. Herein, the chemical potentials of rectangle cavities 1, 2, and 3 are $0.42,0.43$, and $0.44 \mathrm{eV}$, respectively. Fig. 11(a) shows the magnetic field distribution at the peak wavelength of $\lambda_{12}=6232 \mathrm{~nm}$ when the light is resonated between cavities 1 and 2. Moreover, Fig. 11(b) shows the magnetic field distribution at the peak wavelength of $\lambda_{23}=6029 \mathrm{~nm}$ when the light is resonated between cavities 2 and 3. The corresponding transmission spectrum of the dual-PIT effect is shown in Fig. 10(d1). The results indicate that the three rectangle cavities act as three resonators, and the Fabry-Perot resonance is realized between cavities 1 and 2 or cavities 2 and 3. Therefore, the formation mechanism of the dual PIT effect is the same as that of the single PIT effect, which is caused by resonance between two rectangle cavities.

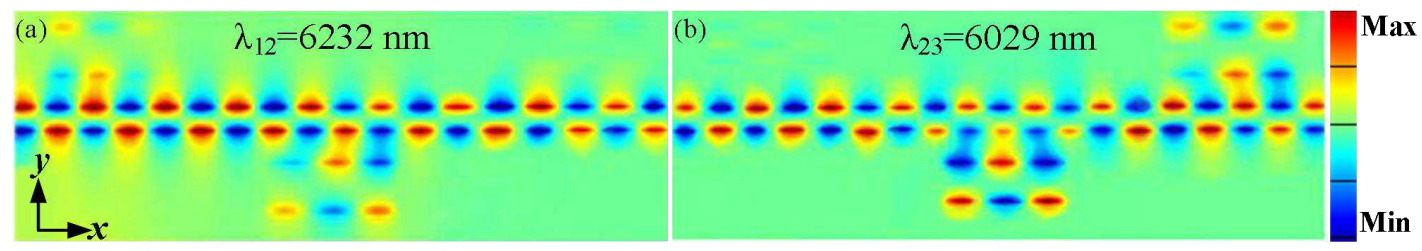

Fig. $11\left|H_{z}\right|^{2}$ magnetic field distributions of the dual-PIT effect in triple cavities side-coupled with a graphene nanoribbon waveguide system at the peak wavelength

\section{Simulation Results And Discussion Of Triple-PIT System}

Fig. 1(d) shows that the triple PIT system comprises rectangle cavities 1, 2, 3, 4, and the graphene nanoribbon waveguide. The triple-PIT effect is also obtained by the combination of a bright and dark mode coupling and the cavities side-coupled with a graphene nanoribbon waveguide mechanism. The chemical potentials of graphene rectangle cavities $1,2,3$, and 4 are initially set to $0.40,0.42,0.42$, and $0.44 \mathrm{eV}$, respectively. The dynamic transmission characteristics for the triple-PIT system in Fig. 12 are analyzed by a temporal CMT. The normalized amplitude $a_{i}$ for the time-harmonic field $e^{-j \omega t}$ of the $i$ th cavity $(i=1,2,3,4)$ are given by the following: 


$$
\begin{aligned}
& \frac{d a_{1}}{d t}=\left(j \omega_{01}-\kappa_{\mathrm{int}, 1}-\kappa_{\mathrm{w}, 1}\right) a_{1}+\sqrt{\kappa_{\mathrm{w}, 1}} S_{+, \mathrm{in}}^{(1)}+\sqrt{\kappa_{\mathrm{w}, 1}} S_{-, \mathrm{in}}^{(1)}-j \mu_{12} a_{2}, \\
& \frac{d a_{2}}{d t}=\left(j \omega_{01}-\kappa_{\mathrm{int}, 2}\right) a_{2}-j \mu_{12} a_{1}, \\
& \frac{d a_{3}}{d t}=\left(j \omega_{02}-\kappa_{\mathrm{int,3}}-\kappa_{\mathrm{w}, 3}\right) a_{3}+\sqrt{\kappa_{\mathrm{w}, 3}} S_{+, \mathrm{in}}^{(2)}+\sqrt{\kappa_{\mathrm{w}, 3}} S_{-, \mathrm{in}}^{(2)}-j \mu_{34} a_{4}, \\
& \frac{d a_{4}}{d t}=\left(j \omega_{02}-\kappa_{\mathrm{int}, 4}\right) a_{4}-j \mu_{34} a_{3},
\end{aligned}
$$

where $\omega_{01}$ is the intrinsic resonant frequency of cavities 1 and 2 , and $\omega_{02}$ is the intrinsic resonant frequency of cavities 3 and $4 . \kappa_{\mathrm{w}, i}(i=1,3)$ is the attenuation ratio due to energy coupling into the graphene nanoribbon waveguide, and $\kappa_{\mathrm{int}, i}(i=1,2,3,4)$ is the attenuation ratio of the $i$ th rectangle cavity. $\mu_{12}=\omega_{01} /\left(2 Q_{\mathrm{c}, 1}\right)$ and $\mu_{34}=\omega_{02} /\left(2 Q_{\mathrm{c}, 2}\right)$ are the coupling coefficients between cavities 1 and 2 and between cavities 3 and 4 , respectively. $Q_{\mathrm{w}, i}(i=1,3), Q_{\mathrm{int}, i}(i=1,2,3,4)$, and $Q_{\mathrm{c}, i}(i=1,2)$ are the waveguide coupling loss, cavity quality factors due to the intrinsic loss, and direct coupling between bright and dark modes, respectively.

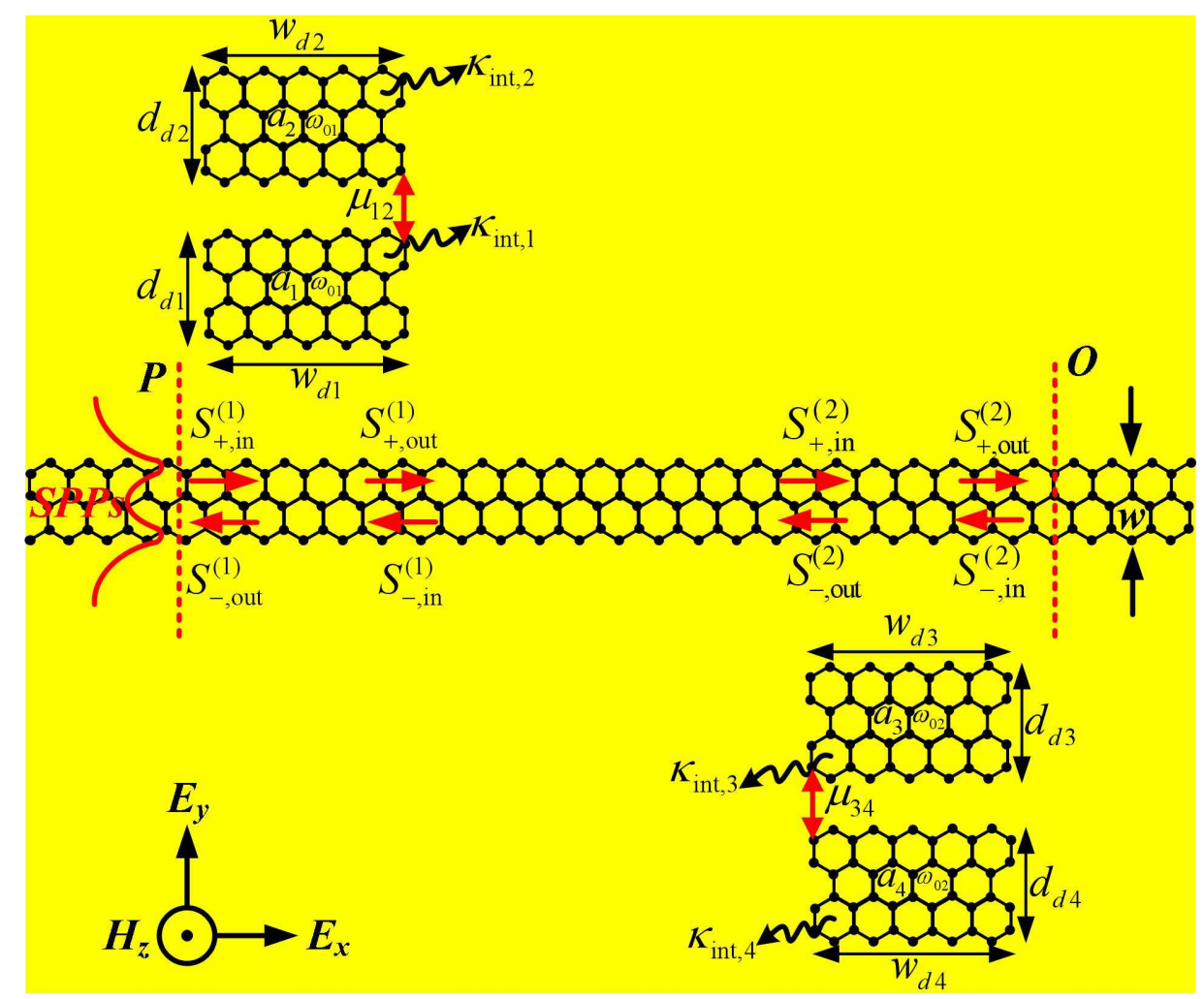

Fig. 12 Schematic of the realizing principle of the triple-PIT system

The energy conservation of the output wave amplitude in each cavity indicates that the output spectral transmittance of the system for the triple-PIT system is as follows: 


$$
T_{4}=\left|t_{d}\right|^{2}=\left|\frac{S_{+, \text {out }}^{(2)}}{S_{+, \text {in }}^{(1)}}\right|^{2}=\left|\frac{\left(\beta_{3}+\frac{\mu_{34}^{2}}{\beta_{4}}\right)\left(\beta_{1}+\frac{\mu_{12}^{2}}{\beta_{2}}\right)}{\left(\beta_{1}+\kappa_{\mathrm{w}, 1}+\frac{\mu_{12}^{2}}{\beta_{2}}\right)\left(\beta_{3}+\kappa_{\mathrm{w}, 3}+\frac{\mu_{34}^{2}}{\beta_{4}}\right) e^{j \phi}-\kappa_{\mathrm{w}, 1} \kappa_{\mathrm{w}, 3} e^{-j \phi}}\right|^{2} .
$$

where $\beta_{1}=j\left(\omega-\omega_{01}\right)+\kappa_{\mathrm{int}, 1}, \beta_{2}=j\left(\omega-\omega_{01}\right)+\kappa_{\mathrm{int}, 2,}, \beta_{3}=j\left(\omega-\omega_{02}\right)+\kappa_{\mathrm{int}, 3}, \beta_{4}=j\left(\omega-\omega_{02}\right)+\kappa_{\mathrm{int}, 4}$, and $t_{d}$ are the transmission coefficients of the triple-PIT system.

The corresponding intrinsic quality factors are $\left(Q_{\mathrm{int}, 1}=119.5,123.1,124.3,127.8,\left(Q_{\mathrm{int}, 2}=116.2\right.\right.$, $122.5,131.2,136.1),\left(Q_{\mathrm{int}, 3}=117.1,119.2,121.7,124.2\right)$, and $\left(Q_{\mathrm{int}, 4}=113.3,119.6,125.6,133.5\right)$ when the chemical potentials of rectangle cavities $1,2,3$, and 4 are $(0.40,0.39,0.42$, and $0.41 \mathrm{eV}),(0.40$, $0.40,0.42$, and $0.42 \mathrm{eV}),(0.40,0.41,0.42$, and $0.43 \mathrm{eV})$, and $(0.40,0.42,0.42$, and $0.44 \mathrm{eV})$. The total quality factors are caused by the triple-PIT transparent peaks, namely, $Q_{\mathrm{t}, 1}=44.36,43.21,42.37,41.50$; $Q_{\mathrm{t}, 3}=47.88,47.04,46.19,45.32$. Hence, the coupling quality factors of rectangle cavities 1 and 3 are $Q_{\mathrm{w}, 1}=71.02,66.57,64.28,61.46$ and $Q_{\mathrm{w}, 3}=81.00,77.71,74.44,71.36$. Herein, $Q_{\mathrm{c}, 1}=42.13,41.85$, 41.27, 40.93, and $Q_{\mathrm{c}, 2}=41.43,41.12,40.87,40.46$.

Fig. 13(d1) shows that peak I is the PIT transparent peak obtained by the bright and dark mode coupling mechanism attributed to the light resonance in cavities 1 and 2 . The amplitude of the output wave in cavities 1 and 2 is equal to that in cavities 3 and 4 because the light is not coupled into cavities 3 and 4. Hence, $S_{+, \text {out }}^{(1)}=S_{+, \text {out }}^{(2)}$, and $T_{4}^{\prime}=\left|\frac{S_{+ \text {,out }}^{(1)}}{S_{+, \text {in }}^{(1)}}\right|^{2}$. Instead, peak II is the PIT transparent peak based on the cavities side-coupled with a graphene nanoribbon waveguide mechanism attributed to the light resonance in cavities 1 and 3. Consequently, $T_{4}^{\prime \prime}=\left|\frac{S_{+, \text {out }}^{(2)}}{S_{+, \text {in }}^{(1)}}\right|^{2}$. Similar to peak I, peak III is the PIT transparent peak based on the mechanism of the bright and dark mode coupling caused by the light resonance in cavities 3 and 4 . The amplitude of the input wave in cavities 1 and 2 is equal to that in cavities 3 and 4 because the light is not coupled into cavities 1 and 2 . Therefore, $S_{+, \text {in }}^{(1)}=S_{+, \text {in }}^{(2)}$, and $T_{4}^{\prime \prime \prime}=\left|\frac{S_{+, \text {out }}^{(2)}}{S_{+, \text {in }}^{(2)}}\right|^{2}$.

Figs. 13(a1)-(d1) show the normalized transmission intensity spectra of triple-PIT effect under various chemical potentials of graphene rectangle cavities. The peak wavelengths show a blue shift, and the triple-PIT transmission peaks increase with the chemical potentials of graphene rectangle cavities, as shown in Figs. 13(a1)-(d1). Fig. 13(a1) shows that the transmission peaks I, II, and III of the triple-PIT effect are the minimum values of $51 \%, 44 \%$, and $51 \%$, respectively, when the chemical potentials of graphene rectangle cavities $1,2,3$, and 4 are $0.40,0.39,0.42$, and $0.41 \mathrm{eV}$, respectively Meanwhile, the values of the transmission peaks I, II, and III can reach $74 \%, 65 \%$, and $73 \%$, respectively. These results are attributed to the strengthened coupling strength, which is affected by the resonance between the bright and dark modes and the resonance between rectangle cavities and a graphene nanoribbon waveguide when the chemical potentials of graphene rectangle cavities 1, 2, 3, 
and 4 are $0.40,0.42,0.42$, and $0.44 \mathrm{eV}$, respectively, as shown in Fig. 13(d1). Therefore, the triple-PIT effect can be effectively tuned by adjusting the chemical potential of the graphene rectangle cavity.
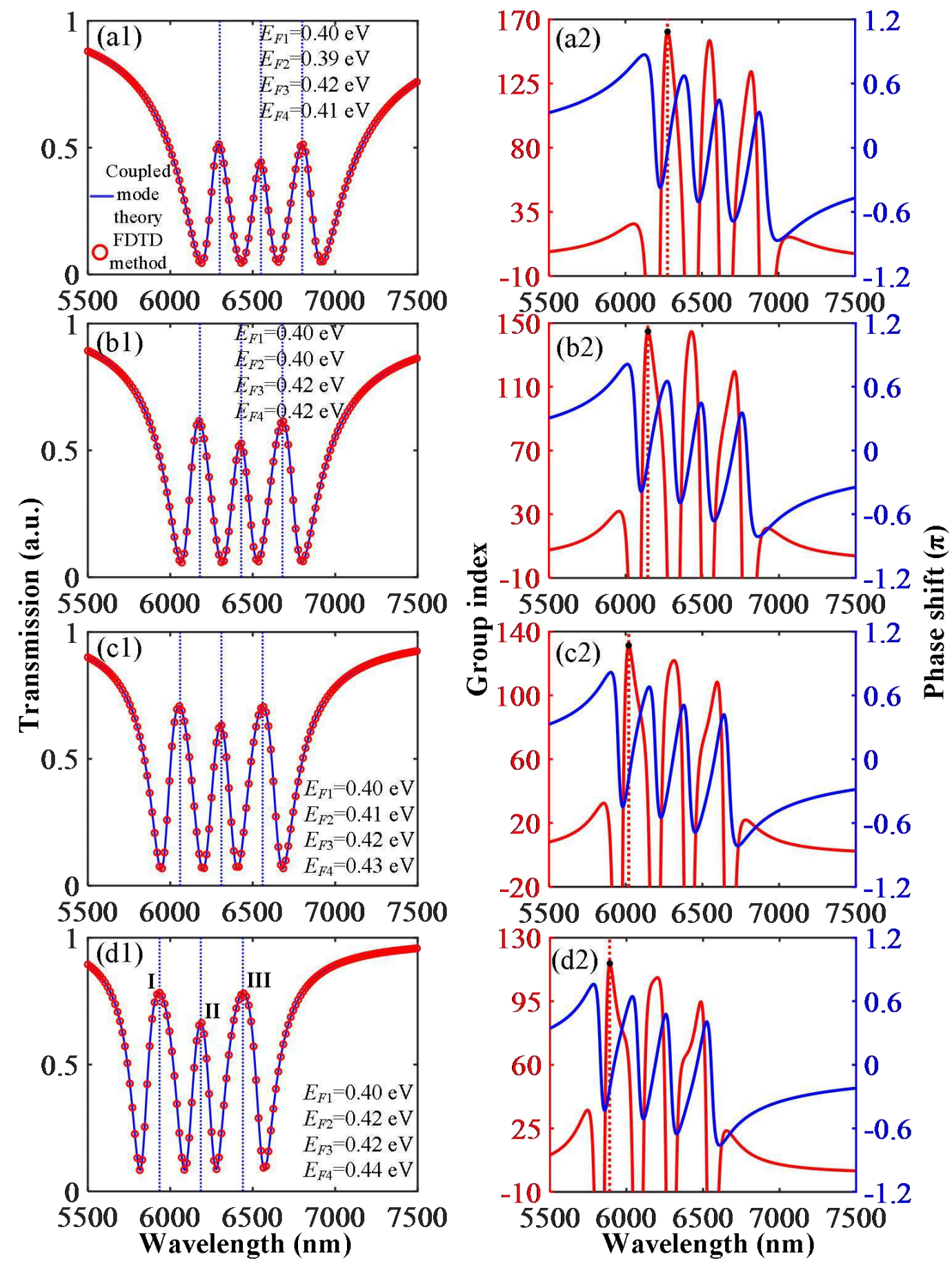

Fig. 13 Triple-PIT effect simulation analysis. a1-d1 Transmission spectra of the triple-PIT effect. a2-d2 Corresponding phase-shift responses and group index

Figs. 13(a2)-(d2) show the transmission spectrum phase shift and group index of triple-PIT effect with the change in the chemical potential of graphene rectangle cavity. The group index sharply drops due to the sudden change in the phase shift at the transmission dip. A $2 \pi$ phase shift exists at the peak wavelength, and the highest group index reaches 161.4. Hence, a large group index is obtained near the transmission dip due to the strong dispersion. The corresponding frequency light signal remains longer than other frequencies when it travels in this structure. This feature enables the device to be used in 
optical storage applications. If the chemical potential of the dark mode of cavities 2 and 4 is tuned to differ from the bright mode of cavities 1 and 3 by varying applied voltage attached to graphene, then the group index increases, and the corresponding maximum group indexes are 161.4, 144.8, 131.4, and 115.8, as shown in the black dots of Figs. 13(a2)-(d2). Thus, the triple-PIT phenomenon can be effectively modulated to seek the desired slow light effect. These results can be applied to dynamically tunable three-channel optical filters [6], optical modulators [38], sensors [39], switching [40], and slow light devices [11] with high transmission, ultranarrow bandwidth, and good slow light effect.

Figs. 14(a)-(c) show the field distributions of $\left|H_{z}\right|^{2}$ at the three peak wavelengths represented by I, II, and III by using the FDTD method in Fig. 13(d1) to further understand the triple-PIT effect mechanism of this structure. The field distributions of $\left|H_{z}\right|^{2}$ shown in Figs. 14(a) and (c) demonstrate that the strongly destructive interference between the bright and dark cavities produced the transmission peaks I and III, thereby enabling the propagation of the SPP wave through the structure without limitation. Notably, the dark cavity is activated strongly, and the magnetic field of the bright cavity is activated weakly at 5935 and $6440 \mathrm{~nm}$. Therefore, the drastic excitation of the dark cavity can destructively restrain the oscillation of the bright cavity. Moreover, the occurrence of the transmission peak II at $6185 \mathrm{~nm}$ is very different from that of peaks I and III because all bright cavities are activated simultaneously, as shown in Fig. 14(b). Meanwhile, the bright-dark mode and waveguide couplings between the cavities below and above the graphene nanoribbon waveguide affect the transmission spectrum simultaneously. The formation of transmission peak II is due to the destructive interference between the magnetic fields of cavities below and above the graphene nanoribbon waveguide.

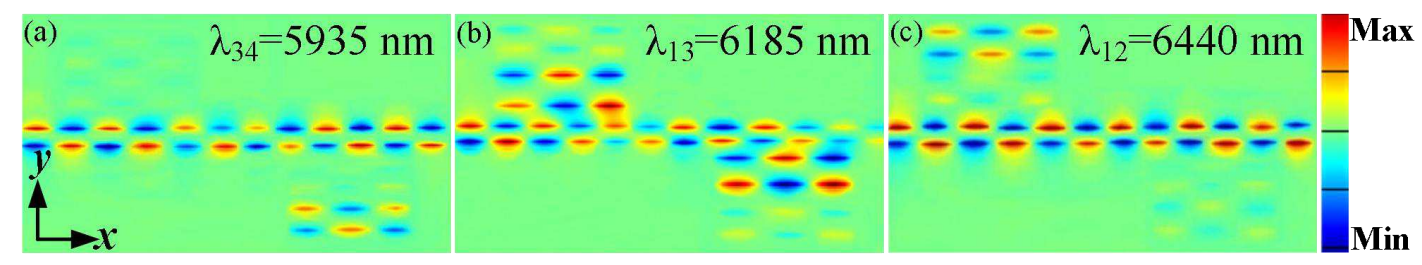

Fig. $14\left|H_{z}\right|^{2}$ magnetic field distributions of the triple-PIT effect at the peak wavelength

Table II shows the maximum transmission and group index of the dual and triple-PIT systems under different chemical potentials of graphene rectangle cavities and pump light intensity at the peak wavelength of PIT. The transmission peak of the dual-PIT effect is tuned from $21 \%$ to $74 \%$ and the group index of the dual PIT system is controlled between 143.2 and 108.6 when the chemical potentials of graphene rectangle cavities 1,2 , and 3 are $0.39 \mathrm{eV}$ to $0.42 \mathrm{eV}, 0.40 \mathrm{eV}$ to $0.43 \mathrm{eV}$, and $0.41 \mathrm{eV}$ to $0.44 \mathrm{eV}$, respectively. This finding indicates that dual PIT systems always maintain a large group index and good slow light effect. Furthermore, the transmission peak of the triple-PIT effect is tuned from $51 \%$ to $74 \%$, and the group index of the triple-PIT system is controlled between 161.4 and 115.8 when the chemical potentials of graphene rectangle cavities 2 and 4 are $0.39 \mathrm{eV}$ to $0.42 \mathrm{eV}$ and $0.41 \mathrm{eV}$ to $0.44 \mathrm{eV}$, respectively. Thus, the maximum group index is realized, and the best slow light effect can be obtained in the triple-PIT system. In addition, Han et al. proposed that the multiband PIT effect is dynamically tuned by a pump light [26]. The maximum transmission of the PIT effect is $58 \%$ when the intensity of the pump light is $5 \mathrm{MW} \cdot \mathrm{cm}^{-2}$, and the group index can reach 6. Dynamically tunable slow light effect is obtained using the pump light. However, a considerably large group index can be 
obtained, and an effective slow light effect can be achieved in the PIT system investigated in this study.

Table II Maximum transmission and group index of the dual- and triple-PIT systems under different chemical potentials of graphene rectangle cavities and pump light intensity at the peak wavelength of PIT

\begin{tabular}{|c|c|c|c|c|}
\hline $\begin{array}{l}\text { Tuning } \\
\text { method }\end{array}$ & System model & Corresponding parameters & $\begin{array}{l}\text { Maximum } \\
\text { transmission } \\
\text { of PIT peak }\end{array}$ & $\begin{array}{c}\text { Maximum } \\
\text { group index } \\
\text { of PIT system }\end{array}$ \\
\hline \multirow{8}{*}{$\begin{array}{l}\text { Graphene } \\
\text { chemical } \\
\text { potential }\end{array}$} & Dual-PIT effect of & $E_{F 1}=0.39 \mathrm{eV}, E_{F 2}=0.40 \mathrm{eV}, E_{F 3}=0.41 \mathrm{eV}$ & $21 \%$ & 143.2 \\
\hline & triple cavities side- & $E_{F 1}=0.40 \mathrm{eV}, E_{F 2}=0.41 \mathrm{eV}, E_{F 3}=0.42 \mathrm{eV}$ & $37 \%$ & 127.3 \\
\hline & coupled with & $E_{F 1}=0.41 \mathrm{eV}, E_{F 2}=0.42 \mathrm{eV}, E_{F 3}=0.43 \mathrm{eV}$ & $50 \%$ & 116.2 \\
\hline & waveguide system & $E_{F 1}=0.42 \mathrm{eV}, E_{F 2}=0.43 \mathrm{eV}, E_{F 3}=0.44 \mathrm{eV}$ & $74 \%$ & 108.6 \\
\hline & \multirow{4}{*}{ Triple-PIT system } & $E_{F 1}=0.40 \mathrm{eV}, E_{F 2}=0.39 \mathrm{eV}, E_{F 3}=0.42 \mathrm{eV}, E_{F 4}=0.41 \mathrm{eV}$ & $51 \%$ & 161.4 \\
\hline & & $E_{F 1}=0.40 \mathrm{eV}, E_{F 2}=0.40 \mathrm{eV}, E_{F 3}=0.42 \mathrm{eV}, E_{F 4}=0.42 \mathrm{eV}$ & $62 \%$ & 144.8 \\
\hline & & $E_{F 1}=0.40 \mathrm{eV}, E_{F 2}=0.41 \mathrm{eV}, E_{F 3}=0.42 \mathrm{eV}, E_{F 4}=0.43 \mathrm{eV}$ & $67 \%$ & 131.4 \\
\hline & & $E_{F 1}=0.40 \mathrm{eV}, E_{F 2}=0.42 \mathrm{eV}, E_{F 3}=0.42 \mathrm{eV}, E_{F 4}=0.44 \mathrm{eV}$ & $74 \%$ & 115.8 \\
\hline $\begin{array}{c}\text { Pump } \\
\text { light } \\
\text { intensity }\end{array}$ & $\begin{array}{l}\text { Triple-PIT effect of } \\
\text { four disk cavities side- } \\
\text { coupled with a } \\
\text { waveguide system }\end{array}$ & $I=5 \mathrm{MW} \cdot \mathrm{cm}^{-2}$ & $58 \%[26]$ & $6[26]$ \\
\hline
\end{tabular}

\section{Conclusions}

In summary, dynamic tunable multiband PIT effects are theoretically and numerically studied on the basis of considerable rectangle cavities coupled with a plasmon waveguide system by tuning the chemical potential of the graphene rectangle cavity. Two completely different realization methods are used to achieve single-PIT effect: one is the bright and dark mode coupling through direct destructive interference, and the other is the cavity coupled with a graphene nanoribbon waveguide through indirect destructive interference. In addition, dual-PIT effect is obtained by three rectangle cavities side-coupled with a graphene nanoribbon waveguide. The transmission of the dual-PIT effect can be significantly tuned between 0.21 and 0.74 , and the corresponding group index is controlled between 143.2 and 108.6. This finding indicates that a large group index is maintained and a good slow light effect is realized in the dual-PIT system. Furthermore, the triple-PIT effect is achieved by combining bright-dark mode coupling and the cavities side-coupled with a graphene nanoribbon waveguide mechanism. The results reveal that considerably sharp transparent windows of triple-PIT effect can be obtained, a high transmission is maintained between 0.51 and 0.74 , and the corresponding group index is controlled between 161.4 and 115.8. Therefore, the maximum group index is realized, and the high transmission and the best slow light effect can be obtained in the triple-PIT system. The proposed structure is ultra-compact because its size is less than $0.5 \mu \mathrm{m}^{2}$ and considerably easier to fabricate than the proposed graphene-based metamaterial structures. Moreover, the slow light effect is more significant than the metal waveguide coupled structure. Therefore, the current study not only introduces a novel approach toward the realization of optical sensors and optical filters, but also has important 
application in slow light and light storage devices with ultrafast, ultracompact, multiband, and dynamic tunable.

\section{Declarations:}

Funding

This work is supported by the National Natural Science Foundation of China (Grant No. 11647122), the Natural Science Foundation of Hubei Provincial (Grant No. 2018CFB672), the Project of the Hubei Provincial Department of Education (Grant Nos. B2021215 and T201617), and the Natural Science Foundation of Xiaogan City (Grant No. XGKJ2021010002).

\section{Competing Interests}

The authors have no relevant financial or non-financial interests to disclose.

\section{Author Contributions}

All authors contributed to the study conception and design. Simulation, numerical calculation, data collection and analysis were performed by Zihao Zhu and Boyun Wang. The first draft of the manuscript was written by Zihao Zhu and Boyun Wang and all authors commented on previous versions of the manuscript. All authors read and approved the final manuscript. Zihao Zhu and Boyun Wang are co-first authors of the article.

\section{Data Availability}

The datasets generated during and analysed during the current study are not publicly available due to science and technology secrecy but are available from the corresponding author on reasonable request.

\section{Ethics approval}

This is a theoretical research. The study do not require ethics approval.

\section{Consent to participate}

Informed consent was obtained from all individual participants included in the study.

\section{Consent to publish}

The authors affirm that consent to publish has been received from all participants.

\section{References}

1. Yang X, Yu M, Kwong D-L, Wong CW (2009) All-optical analog to electromagnetically induced transparency in multiple coupled photonic crystal cavities. Phys Rev Lett 102:173902

2. Harris SE (1997) Electromagnetically induced transparency. Phys Today 50:36-42

3. Hau LV, Harris SE, Dutton Z, Behroozi CH (1999) Light speed reduction to 17 metres per second in an ultracold atomic gas. Nature 397:594-598

4. Liu JH, Yu YF, Zhang ZM (2019) Nonreciprocal transmission and fast-slow light effects in a cavity optomechanical 
system. Opt Express 27:15382-15390

5. Longdell JJ, Fraval E, Sellars MJ, Manson NB (2005) Stopped light with storage times greater than one second using electromagnetically induced transparency in a solid. Phys Rev Lett 95:063601

6. Li H, Wang L, Liu J, Huang Z, Sun B, Zhai X (2013) Investigation of the graphene based planar plasmonic filters. Appl Phys Lett 103:211104

7. Fan CZ, Jia YL, Ren PW, Jia W (2021) Tunable plasmon induced transparency and multispectral transparency with large group delay in graphene metamaterials. J Phys D Appl Phys 54:035107

8. Kekatpure RD, Barnard ES, Cai W, Brongersma ML (2010) Phase-coupled plasmon-induced transparency. Phys Rev Lett 104:243902

9. Zhou L, Ye T, Chen J (2011) Coherent interference induced transparency in self-coupled optical waveguide-based resonators. Opt Lett 36:13-15

10. Zhang X, Liu ZM, Zhang ZB, Gao ED, Luo X, Zhou FQ, Li HJ, Yi Z (2020) Polarization-sensitive triple plasmoninduced transparency with synchronous and asynchronous switching based on monolayer graphene metamaterials. Opt Express 28:36771-36783

11. Ge JH, You CL, Feng H, Li XM, Wang M, Dong LF, Veronis G, Yun MJ (2020) Tunable dual plasmon-induced transparency based on a monolayer graphene metamaterial and its terahertz sensing performance. Opt Express 28:31781-31795

12. Liu N, Langguth L, Weiss T, Kästel J, Fleischhauer M, Pfau T, Giessen H (2009) Plasmonic analogue of electromagnetically induced transparency at the Drude damping limit. Nature Mater 8:758-762

13. Zhang S, Genov DA, Wang Y, Liu M, Zhang X (2008) Plasmon-induced transparency in metamaterials. Phys Rev Lett 101:047401

14. Zhan SP, Kong D, Cao GT, He ZH, Wang Y, Xu GJ, Li HJ (2013) Analogy of plasmon induced transparency in detuned U-resonators coupling to MDM plasmonic waveguide. Solid State Commun 174:50-54

15. Wang BY, Zeng QD, Xiao SY, Xu C, Xiong LB, Lv H, Du J, Yu HQ (2017) Low-power, ultrafast, and dynamic alloptical tunable plasmon induced transparency in two stub resonators side-coupled with a plasmonic waveguide system. J Phys D Appl Phys 50:455107

16. Lai G, Liang RS, Zhang YJ, Bian ZY, Yi LX, Zhan GZ, Zhao RT (2015) Double plasmonic nanodisks design for electromagnetically induced transparency and slow light. Opt Express 23:6554-6561

17. Zhang T, Zhou JZ, Dai J, Dai YT, Han X, Li JQ, Yin FF, Zhou Y, Xu K (2018) Plasmon induced absorption in a graphene-based nanoribbon waveguide system and its applications in logic gate and sensor. J Phys D Appl Phys 51:055103

18. Li HJ, Wang LL, Zhai X (2016) Plasmonically induced absorption and transparency based on MIM waveguides with concentric nanorings. IEEE Photon Technol Lett 28:1454-1457

19. He ZH, Ren XC, Bai SM, Li HJ, Cao DM, Li G (2018) $\Lambda$-Type and V-Type plasmon-induced transparency in plasmonic waveguide systems. Plasmonics 13:2255

20. Xu H, Zhao MZ, Chen ZQ, Zheng MF, Xiong CX, Zhang BH, Li HJ (2018) Sensing analysis based on tunable Fano resonance in terahertz graphene-layered metamaterials. J Appl Phys 123:203103

21. Zhang BH, Li HJ, Xu H, Zhao MZ, Xiong CX, Liu C, Wu K (2019) Absorption and slow-light analysis based on tunable plasmon-induced transparency in patterned graphene metamaterial. Opt Express 27:3598-3608

22. Gao ED, Liu ZM, Li HJ, Xu H, Zhang ZB, Luo X, Xiong CX, Liu C, Zhang BH, Zhou FQ (2019) Dynamically tunable dual plasmon-induced transparency and absorption based on a single-layer patterned graphene metamaterial. Opt Express 27:13884-13894

23. Sun C, Si JN, Dong ZW, Deng XX (2016) Tunable multispectral plasmon induced transparency based on graphene metamaterials. Opt Express 24:11466-11474

24. Chai Z, Hu X, Yang H, Gong Q (2016) All-optical tunable on-chip plasmon-induced transparency based on two surface-plasmon-polaritons absorption. Appl Phys Lett 108:151104

25. Zhan S, Li H, Cao G, He Z, Li B, Yang H (2014) Slow light based on plasmon-induced transparency in dual-ring 
resonator-coupled MDM waveguide system. J Phys D Appl Phys 47:205101

26. Han X, Wang T, Li XM, Liu B, He Y, Tang J (2015) Dynamically tunable slow light based on plasmon induced transparency in disk resonators coupled MDM waveguide system. J Phys D Appl Phys 48:235102

27. Zhu Y, Hu X, Fu Y, Yang H, Gong Q (2013) Ultralow-power and ultrafast all-optical tunable plasmon-induced transparency in metamaterials at optical communication range. Sci Rep 3:2338

28. Han X, Wang T, Li XM, Liu B, He Y, Tang J (2015) Ultrafast and low-power dynamically tunable plasmon-induced transparencies in compact aperture-coupled rectangular resonators. J Lightwave Technol 33:3083-3090

29. Celis A, Nair M, Taleb-Ibrahimi A, Conrad E, Berger C, Heer W, Tejeda A (2016) Graphene nanoribbons: fabrication, properties and devices. J Phys D Appl Phys 49:143001

30. Wang L, Li W, Jiang XY (2015) Tunable control of electromagnetically induced transparency analogue in a compact graphene-based waveguide. Opt Lett 40:2325-2328

31. Noual A, Amrani M, Boudouti EHE, Pennec Y, Djafari-Rouhani D (2019) Plasmon induced transparency in graphene-based side coupled two rectangular nano-cavities-like structures. Materials Today: Proceedings 13:10761083

32. Zhao HL, Ren Y, Fang L, Lin H (2020) Electromagnetic induced transparency in graphene waveguide structure for Terahertz application. Results in Physics 16:102971

33. Li F, Jackson SD, Grillet C, Magi E, Hudson D, Madden SJ, Moghe Y, Brien CO, Read A, Duvall SG, Atanackovic P, Eggleton BJ, Moss DJ (2011) Low propagation loss silicon-on-sapphire waveguides for the mid-infrared. Opt Express 19:15212-15220

34. Xu H, Zhao MZ, Zheng MF, Xiong CX, Zhang BH, Peng YY, Li HJ (2019) Dual plasmon-induced transparency and slow light effect in monolayer graphene structure with rectangle defects. J Phys D Appl Phys 52:025104

35. Xu H, Li H, He Z, Chen Z, Zheng M, Zhao M (2017) Dual tunable plasmon-induced transparency based on siliconair grating coupled graphene structure in terahertz metamaterial. Opt Express 25:20780-20790

36. Wei BZ, Jian SS (2017) Analogue of electromagnetically-induced transparency based on graphene nanotube waveguide. J Phys D Appl Phys 50:355101

37. Wang T, Zhang Y, Hong Z, Han Z (2014) Analogue of electromagnetically induced transparency in integrated plasmonics with radiative and subradiant resonators. Opt Express 22:21529-21534

38. Ren TX, Chen L (2019) Slow light enabled high-modulation-depth graphene modulator with plasmonic metasurfaces. Opt Lett 44:5446-5449

39. Neubert TJ, Wehrhold M, Kaya NS, Balasubramanian K (2020) Faradaic effects in electrochemically gated graphene sensors in the presence of redox active molecules. Nanotechnology 31:405201

40. Zhou FQ, Wang YQ, Zhang X, Wang JW, Liu ZM, Luo X, Zhang ZB, Gao ED (2021) Dynamically adjustable plasmon-induced transparency and switching application based on bilayer graphene metamaterials. J Phys D Appl Phys 54:054002 